\title{
Effect of nitrogen seeding on the energy losses and on the time scales of the electron temperature and density collapse of Type I ELMs in JET with the ITER-like wall
}

\author{
L. Frassinetti ${ }^{1}$, D. Dodt ${ }^{2}$, M.N.A. Beurskens ${ }^{3}$, A Sirinelli ${ }^{3}$, J. E. Boom ${ }^{2}$, T. Eich ${ }^{2}$, J. Flanagan ${ }^{3}$, \\ C. Giroud ${ }^{3}$, M. S. Jachmich ${ }^{3}$, M. Kempenaars ${ }^{3}$, P. Lomas ${ }^{3}$, G. Maddison ${ }^{3}$, C. Maggi ${ }^{2}$, R. \\ $\mathrm{Neu}^{2,4}$, I. Nunes ${ }^{5}$, C. Perez von Thun ${ }^{2}$, B. Sieglin ${ }^{2}$, M.Stamp ${ }^{3}$ and JET-EFDA contributors*
}

JET-EFDA, Culham Science Centre, Abingdon, OX14 3DB, UK

${ }^{1}$ Division of Fusion Plasma Physics, Association EURATOM-VR , KTH, SE-10044 Stockholm, Sweden

${ }^{2}$ Max-Planck-Institut für Plasma Physik, Boltzmannstr.2, 85748 Garching, Germany

${ }^{3}$ EURATOM /CCFE Fusion Association, Culham Science Centre, Abingdon, OX14 3DB, UK

${ }^{4}$ Technische Universität München, Boltzmannstr. 15, 85748 Garching, Germany

${ }^{5}$ Centro de Fusao Nuclear, Associacao EURATOM-IST, Lisboa, Portugal

*See appendix of F. Romanelli et al., Fusion Energy, 2012 (24th IAEA Intern. Conference, S.

Diego, 2012).

\begin{abstract}
The baseline type I ELMy H-mode scenario has been re-established in JET with the new tungsten MKII-HD divertor and beryllium on the main wall (hereafter called ITER-like wall, JET-ILW).

The first JET-ILW results show that the confinement is degraded by $20-30 \%$ in the baseline scenarios compared to the previous carbon wall JET (JET-C) plasmas. The degradation is mainly driven by the reduction in the pedestal temperature. Stored energies and pedestal temperature comparable to the JET-C have been obtained to date in JET-ILW baseline plasmas only in the high triangularity shape using $\mathrm{N}_{2}$ seeding.

This work compares the energy losses during ELMs and the corresponding time scales of the temperature and density collapse in JET-ILW baseline plasmas with and without $\mathrm{N}_{2}$ seeding with similar JET-C baseline plasmas. ELMs in the JET-ILW differ from those with the carbon wall both in terms of time scales and energy losses. The ELM time scale, defined as the time to reach the minimum pedestal temperature soon after the ELM collapse, is $\approx 2 \mathrm{~ms}$ in the JETILW and lower than $1 \mathrm{~ms}$ in the JET-C. The energy losses are in the range $\Delta \mathrm{W}_{\text {ELM }} / \mathrm{W}_{\text {ped }} \approx 7$ $12 \%$ in the JET-ILW and $\Delta \mathrm{W}_{\mathrm{ELM}} / \mathrm{W}_{\text {ped }} \approx 10-20 \%$ in JET-C, and fit relatively well with earlier multi-machine empirical scalings of $\Delta \mathrm{W}_{\mathrm{ELM}} / \mathrm{W}_{\text {ped }}$ with collisionality. The time scale of the ELM collapse seems to be related to the pedestal collisionality. Most of the non-seeded JETILW ELMs are followed by a further energy drop characterized by a slower time scale $\approx 8$ $10 \mathrm{~ms}$ (hereafter called slow transport events), that can lead to losses in the range $\Delta \mathrm{W}_{\text {slow }} / \mathrm{W}_{\text {ped }} \approx 15-22 \%$, slightly larger than the losses in JET-C. The $\mathrm{N}_{2}$ seeding in JET-ILW significantly affects the ELMs. The JET-ILW plasmas with $\mathrm{N}_{2}$ seeding are characterized by ELM energy losses and time scales similar to the JET-C and by the absence of the slow transport events.
\end{abstract}




\section{INTRODUCTION}

Tokamak plasmas in the H-mode regime are characterized by periodic edge localized modes (ELMs) that produce the partial collapse of the pedestal pressure and the consequent release of energy and particles towards the wall and the divertor targets. Material studies $[1,2,3]$ have shown that in ITER the maximum tolerable ELM energy loss $\left(\Delta \mathrm{W}_{\mathrm{ELM}}\right)$ is approximately $1 \mathrm{MJ}$ for the baseline $\mathrm{Q}_{\mathrm{DT}}=10$ ITER scenario, corresponding to approximately $1 \%$ of the ITER pedestal stored energy ( $\left.\mathrm{W}_{\text {ped }}\right)$. A multi-device study performed on DIII-D, ASDEX, JT60U and on the carbon wall JET (hereafter JET-C) has shown that the relative ELM energy losses scale with the inverse of the pedestal collisionality [4] predicting large losses for the ITER target collisionality with $\Delta \mathrm{W}_{\mathrm{ELM}} / \mathrm{W}_{\text {ped }}>15 \%$.

To reduce the long term tritium retention, ITER will not operate with a carbon wall but with beryllium in the main chamber and tungsten in the divertor. In order to study the effects of the plasma on these materials and to improve the prediction for ITER, the carbon wall in JET-C has been replaced in 2010 by an ITER-like wall (JET-ILW) with mostly Be in the main chamber and $\mathrm{W}$ in the divertor $[5,6]$. ASDEX Upgrade operates with a DEMO-relevant full W wall since approximately 2007 [7].

The initial results obtained in the JET-ILW have shown that the first wall material seems to affect both the plasma confinement and the pedestal properties especially in the ITER baseline scenarios $[8,9]$. The plasma confinement factor $\mathrm{H}_{98}$ (defined as the ratio between the energy confinement and the confinement expected from a multi-machine scaling [10]) to achieve the ITER baseline scenarios is $\mathrm{H}_{98}=1$ in the type-I ELMy $\mathrm{H}$-mode plasma. The JET-C has achieved $\mathrm{H}_{98}=1$ both in a low and a high triangularity plasma shape [11, 12, 13], but with the high shape characterized by a better confinement at a given Greenwald density due to the improved pedestal stability. To date, the JET-ILW standard baseline plasma has not routinely achieved a confinement factor $\mathrm{H}_{98}=1$, but is characterized by a confinement factor in the range $\mathrm{H}_{98}=0.8-0.9$ for both low and high triangularity with no significant confinement improvement for the high shape plasmas. The degraded confinement is mainly driven by a lower pedestal pressure due to a pedestal temperature approximately 20-30\% lower than in JET-C. The pedestal density is instead comparable among JET-C and JET-ILW. To date, a JET-ILW pedestal pressure comparable to the baseline JET-C has been achieved only in high triangularity experiments with $\mathrm{N}_{2}$ seeding $[9,14]$. JET-C plasmas with $\mathrm{N}_{2}$ seeding have shown no confinement improvement compared to the non-seeded JET-C plasmas [15].

The metal wall has produced confinement degradation also in ASDEX Upgrade with a partial recovery of the confinement obtained with $\mathrm{N}_{2}$ seeding [16]. As discussed in detail in [17], the confinement degradation with a metal wall and its partial recovery with $\mathrm{N}_{2}$ cannot be ascribed to a core effect, as the core temperature gradient lengths are comparable and can be only partially ascribed to a pedestal ion-dilution effect due to the change of the impurities in the plasma. The mechanism that regulates the pedestal confinement degradation with a metal wall and its partial recovery with $\mathrm{N}_{2}$ seeding is still under investigation. Studies on the ELM dynamics in ASDEX Upgrade with and without $\mathrm{N}_{2}$ seeding are described in [18].

The aim of this work is to characterize the ELM energy losses and the time scales of the electron pedestal temperature $\left(\mathrm{T}_{\mathrm{e}}{ }^{\text {ped }}\right)$ and density $\left(\mathrm{n}_{\mathrm{e}}{ }^{\text {ped }}\right)$ collapses in the non-seeded JETILW plasmas and in the nitrogen seeded JET-ILW plasmas and to compare the results with similar JET-C plasmas.

The paper is organized as follows. Section 2 describes the data set used in this work and the corresponding $\mathrm{T}_{\mathrm{e}}^{\text {ped }}$ and $\mathrm{n}_{\mathrm{e}}{ }^{\text {ped }}$ range during the pre-ELM phase, discusses the ELM type and briefly describes the main diagnotics used. Section 3 describes the pedestal time evolution during the ELM collapse for three representative shots and then quantifies the time scale of the ELM collapse for the entire data set. Section 4 describes the $T_{e}$ and $n_{e}$ profiles before and after the ELMs. Section 5 compares the ELM energy losses considering both the conductive 
and the convective channels. The ELMs in the non-seeded JET-ILW are sometimes followed by a further energy collapse characterized by a time scale slower than the standard ELM. The presence of these events (called "slow transport events") and their energy losses are discussed in Section 6. Finally, Section 7 discusses the results in light of the previous multi-machine collisionality scaling [4] and Section 8 presents the conclusions.

\section{DESCRIPTION OF THE DATABASE}

\subsection{Plasma scenario}

The experimental sessions with $\mathrm{N}_{2}$ seeding in JET-ILW have been performed with plasma current 2.5MA, magnetic field 2.7T and NBI power 15-18MW. The non-seeded plasma analysed in this work have been selected in order to have current, field, power and main gas flow similar to the seeded shots. The main gas $\left(\mathrm{D}_{2}\right)$ flow is in the range $\Gamma_{\mathrm{D} 2}=2-2.510^{22}(\mathrm{e} / \mathrm{s})$ for the high triangularity plasmas and $\Gamma_{\mathrm{D} 2}=0.8-1.610^{22}(\mathrm{e} / \mathrm{s})$ for the low triangularity plasmas. However, for the high triangularity case, these $\Gamma_{\mathrm{D} 2}$ levels are larger than those typically used in JET-C, therefore a further set of high- $\delta$ JET-C shots with the typical fuelling level $\left(\Gamma_{\mathrm{D} 2} \approx 0.3\right.$ $10^{22} \mathrm{e} / \mathrm{s}$ ) is considered. The nitrogen rate in the seeded JET-ILW plasmas is different from shot to shot and is in the range $\Gamma_{\mathrm{N} 2}=0.5-3 \cdot 10^{22}(\mathrm{e} / \mathrm{s})$. Some of the main plasma parameters for the seven groups of shots are summarized in Table 1.

\begin{tabular}{lllllllll}
\hline & $\boldsymbol{\delta}$ & $\mathbf{I p}(\mathbf{k A})$ & $\mathbf{B}(\mathbf{T})$ & $\mathbf{P}_{\mathrm{NBI}}(\mathbf{M W})$ & $\Gamma_{\mathbf{D} 2} \mathbf{1 0}^{22}(\mathbf{e} / \mathbf{s})$ & $\mathbf{f}_{\mathrm{ELM}}(\mathbf{H z})$ & $\mathbf{n}_{\mathbf{e}} / \mathbf{n}_{\mathbf{G W}}$ & $\mathbf{E L M}$ \\
\hline ILW & 0.4 & 2.5 & 2.7 & $15-18$ & $2.5 \pm 0.8$ & $28 \pm 14$ & $0.78 \pm 0.04$ & type I \\
ILW+N2 & 0.4 & 2.5 & 2.7 & $15-18$ & $2.1 \pm 0.9$ & $40 \pm 15$ & $0.92 \pm 0.06$ & type I \\
CFC & 0.4 & 2.5 & 2.7 & $15-18$ & $2.3 \pm 0.5$ & $10 \pm 3$ & $0.92 \pm 0.06$ & type I/II \\
CFC (low gas) & 0.4 & 2.5 & 2.7 & $15-18$ & $0.3 \pm 0.1$ & $22 \pm 3$ & $0.71 \pm 0.04$ & type I \\
ILW & 0.2 & 2.5 & 2.7 & $15-18$ & $1.6 \pm 0.7$ & $33 \pm 15$ & $0.73 \pm 0.03$ & type I \\
ILW+N2 & 0.2 & 2.5 & 2.7 & $15-18$ & $1.1 \pm 0.3$ & $35 \pm 6$ & $0.56 \pm 0.03$ & type I \\
CFC & 0.2 & 2.5 & 2.7 & $15-18$ & $0.8 \pm 0.5$ & $33 \pm 11$ & $0.67 \pm 0.03$ & type I \\
\hline
\end{tabular}

Table 1. Triangularity, current, magnetic field, input power, $D_{2}$ gas rate, ELM frequency, pedestal density relative to the Greenwald density and ELM type of the shots analysed.

\subsection{ELM Type}

Type-I ELMy H-modes are characterized by the increase of the ELM frequency ( $f_{\text {ELM }}$ ) with the input power [19] and by relatively large energy losses $\left(\Delta \mathrm{W}_{\mathrm{ELM}} / \mathrm{W}_{\text {tot }}>1 \%\right)$, while Type-III ELMy H-modes are characterized by the $\mathrm{f}_{\mathrm{ELM}}$ reduction with the input power and by relatively small energy losses. JET-C plasmas also exhibit Type I/II ELMs [12, 20]. A pure Type II regime is classified by the absence of large transient heat loads and by the absence of type I ELMs, producing a continuous heat load on the divertor region. Pure type II ELMs have been observed in ASDEX-Upgrade [21], but in JET only a mixed type I/II regime has been observed. The transition to a mixed type I/II ELMy H-mode can occur at high pedestal density ( $\mathrm{n}_{\mathrm{e}} \geq 0.7-0.8 \mathrm{n}_{\mathrm{GW}}$ ), and is accompanied by a reduction of the ELM frequency compared to the pure type I ELMs.

The JET-C plasmas studied in this work are in the Type-I ELM regime excluding those with high gas flow $\left(\Gamma_{\mathrm{D} 2} \approx 2-2.510^{22}\right)$. The JET-C plasmas with high gas flow have an ELM frequency $\left(\mathrm{f}_{\mathrm{ELM}} \approx 10 \mathrm{~Hz}\right)$ lower than the corresponding low gas JET-C plasmas $\left(\mathrm{f}_{\mathrm{ELM}} \approx 22 \mathrm{~Hz}\right)$, therefore suggesting a mixed type I/II regime. 
The JET-ILW plasmas are in the Type-I ELM regime [9]. In fact, the results presented in the gas scan described in [8,22] show that the threshold between Type I and Type III ELMs is approximately $\mathrm{T}_{\mathrm{e}}{ }^{\text {ped }} \approx 300 \mathrm{eV}$ in the JET-ILW, below the pre-ELM pedestal temperature of the present JET-ILW data $\left(\mathrm{T}_{\mathrm{e}}{ }^{\text {ped }}>500 \mathrm{eV}\right.$, see figure 1$)$.

\subsection{Diagnostics}

The temperature data analysed in this work are measured by the electron cyclotron emission (ECE) radiometer [23] with a time resolution up to $4 \mu \mathrm{s}$. In this work, the radial position of the ECE data has been calculated at each time step using the magnetic field from the fast EFIT equilibrium and then mapped on $\rho_{\theta}$ (the square root of the poloidal flux). As described in detail in reference [24], a correction factor in the vacuum magnetic field is necessary to match the ECE temperature profiles with those measured from the High Resolution Thomson Scattering (HRTS). For the plasmas analysed in this work, the correction factor is less than $1 \%$. The density data are measured by the reflectometer with a time resolution up to $15 \mu \mathrm{s}$ [25]. The radial position of the reflectometer data have been mapped at each time step on $\rho_{\theta}$ using the fast EFIT equilibrium.

When ECE radiometer and/or reflectomer are not available (in particular, the reflectometer data are available only for two JET-C shots), the High Resolution Thomson Scattering (HRTS) [26,27] is used to calculate the energy losses. Due to the low sampling frequency $(20 \mathrm{~Hz})$ of HRTS, the time scale of the ELM collapse is calculated using only ECE and reflectemoter.

\subsection{Pedestal temperature and density}

The pedestal heights for $\mathrm{T}_{\mathrm{e}}{ }^{\text {ped }}$ and $\mathrm{n}_{\mathrm{e}}{ }^{\text {ped }}$ are shown in figure 1 . The pedestal heights are calculated using the HRTS by selecting the profiles in the pre-ELM phase (within the $70 \%$ to 99\% time interval of the ELM cycle).

The JET-C plasmas have pedestal temperature higher than $900 \mathrm{eV}$, and electron pedestal pressure in the range $8-10 \mathrm{kPa}$ for the low- $\delta$ and $10-14 \mathrm{kPa}$ for the high delta- $\delta$. The JET-ILW plasmas without $\mathrm{N}_{2}$ seeding (red symbols) are characterized by a pedestal density in the range 6-8 $10^{19} \mathrm{~m}^{-3}$, comparable to the JET-C data, but by $\mathrm{T}_{\mathrm{e}}{ }^{\text {ped }} \approx 500-720 \mathrm{eV}$, which is approximately $20 \%$ lower than the coldest JET-C plasma considered. When $\mathrm{N}_{2}$ is seeded into high- $\delta$ JETILW plasmas (green triangles), higher $\mathrm{n}_{\mathrm{e}}^{\text {ped }}$ and $\mathrm{T}_{\mathrm{e}}{ }^{\text {ped }}$ can be reached with an electron pedestal pressure up to $11 \mathrm{kPa}$, which is comparable to the JET-C plasmas. As discussed in reference [9], the rate of confinement improvement is dependent on the level of $\mathrm{N}_{2}$ gas injected. This behaviour is significantly different for the low- $\delta$ JET-ILW plasmas. In this case, the seeding produces a slight reduction of the pedestal density and a slight increase of the pedestal temperature, with the pedestal pressure that remains unaffected.

Concerning the ion temperature $T_{i}$, it has been verified $T_{e}=T_{i}$ for the JET-C shots. The JETILW shots with available $T_{i}$ measurement have $T_{e}=T_{i}$ as well. All the JET-ILW shots have high collisionality [8], so a difference between $T_{e}$ and $T_{i}$ is not expected. 


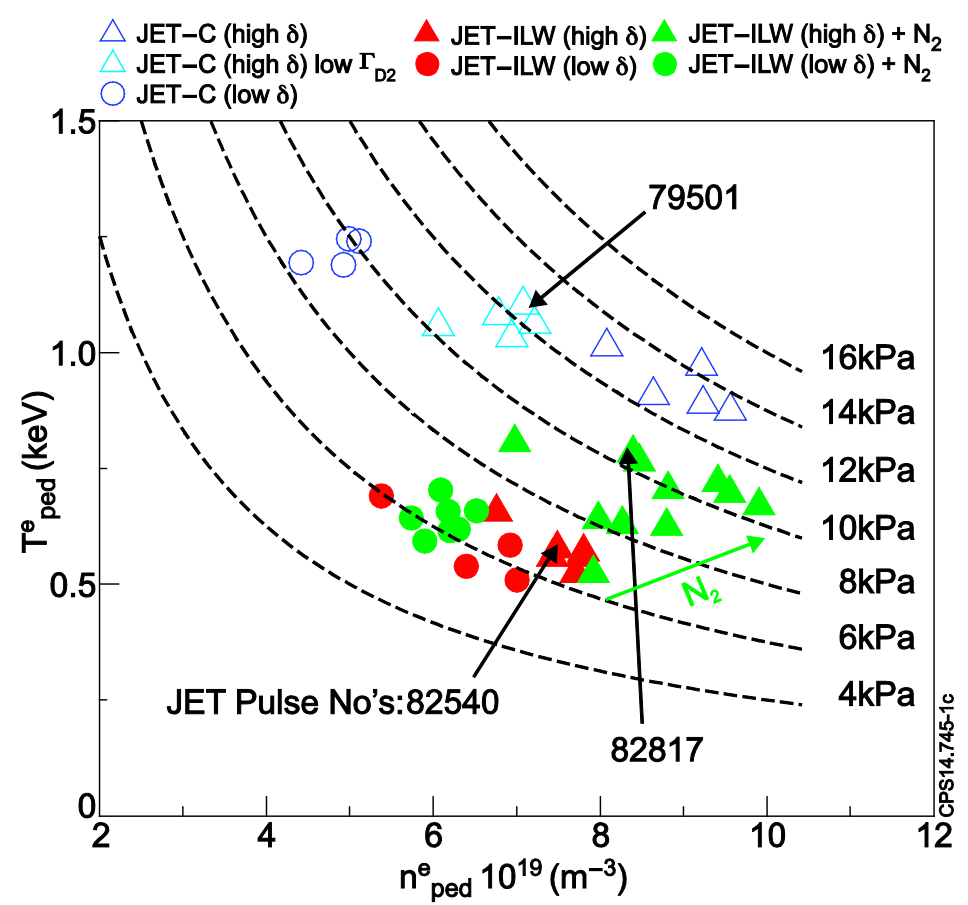

Figure 1. Electron pedestal temperature and density for JET-C (empty symbols) and JET-ILW (full symbols) plasmas in the pre-ELM phase. JET-ILW plasmas with $\mathrm{N}_{2}$ seeding are in green. $H i g h-\delta$ shots are represented with triangles and low- $\delta$ with circles. The dashed lines highlight the corresponding electron pedestal pressure. The green arrow highlights the increase of $\mathrm{N}_{2}$ flow.

\section{TIME SCALE OF THE ELM COLLAPSE}

The time evolutions of electron temperature and density at the top of the pedestal for three high- $\delta$ shots (a JET-C plasma, a non-seeded JET-ILW plasma and a JET-ILW plasma with $\mathrm{N}_{2}$ seeding) are shown in figure 2. The temperature data are measured by the ECE radiometer and the density data by the reflectometer.

The ELM effect is evident by the drop in the electron temperature and density. The temperature and density drops are approximately $\Delta \mathrm{T}_{\mathrm{e}} \approx 350 \mathrm{eV}$ and $\Delta \mathrm{n}_{\mathrm{e}} \approx 1 \cdot 10^{19} \mathrm{~m}^{-3}$ for the JETC shot, $\Delta \mathrm{T}_{\mathrm{e}} \approx 150 \mathrm{eV}$ and $\Delta \mathrm{n}_{\mathrm{e}} \approx 1.5 \cdot 10^{19} \mathrm{~m}^{-3}$ for the JET-ILW shot without $\mathrm{N} 2$ seeding. For the JET-ILW shot with N2 seeding the drops are between the JET-C shot and the JET-ILW shot.

The two ELMs of the non-seeded JET-ILW shot (second column in figure 2) are significantly different. For the first ELM $(t \approx 16.38 \mathrm{~s})$ the electron temperature starts to recover soon after the collapse, while the $T_{e}$ collapse for the second ELM $(t \approx 16.43 \mathrm{~s})$ is followed by a further $T_{e}$ reduction characterized by a slower decay rate. A different behaviour of the two ELMs is present also in density, in the high frequency MHD activity and in the Beryllium signal, figures 2(f), 2(g) and 2(h). The high frequency MHD activity is calculated by integrating the spectrogram of a magnetic pick-up coil in the frequency range $40-200 \mathrm{kHz}$. The MHD activity and the Be signal have an initial sharp peak for both ELMs. During the second ELM, the sharp peak is followed by a $\approx 10 \mathrm{~ms}$ long time interval during which the signals are higher than their pre-ELM phase. The second phase that follows the $t \approx 16.43 \mathrm{~s}$ ELM is hereafter called "slow transport event". The behaviour of the slow transport event shown in figure 2, and in particular the MHD activity, is similar to what observed in ASDEX Upgrade without $\mathrm{N}_{2}$ seeding during the ELM phase labelled " $1 b$ " [18]. A discussion on the comparison between the "slow transport event" in JET-ILW and the " $1 b$ " phase in ASDEX Upgrade ELMs is presented in Section 7. 
The difference between a collapse characterized by an ELM and a collapse characterized by an ELM followed by a slow transport event is further investigated in figure 3, where the $\tau_{\min }$ distribution is shown. $\tau_{\min }$ is defined as the time to reach the minimum pedestal $T_{e}$ for each ELM collapse. In figure $3, \tau_{\min }$ has been calculated for all the collapses of the high- $\delta$ JETILW shots of the present data set with no $\mathrm{N}_{2}$ seeding. The distribution has two clear peaks. The data with $\tau_{\min }<4 \mathrm{~ms}$ correspond to the collapses due to ELMs only (and correspond to the data used to quantify the time scale of the ELM collapse, $\tau_{\mathrm{ELM}}$, in figures 4 and 5). The data with $\tau_{\min }>4 \mathrm{~ms}$ correspond to the collapses due to an ELM followed by a slow transport event. It is possible to clearly identify if a collapse if followed by a slow transport event by estimating $\tau_{\min }$.
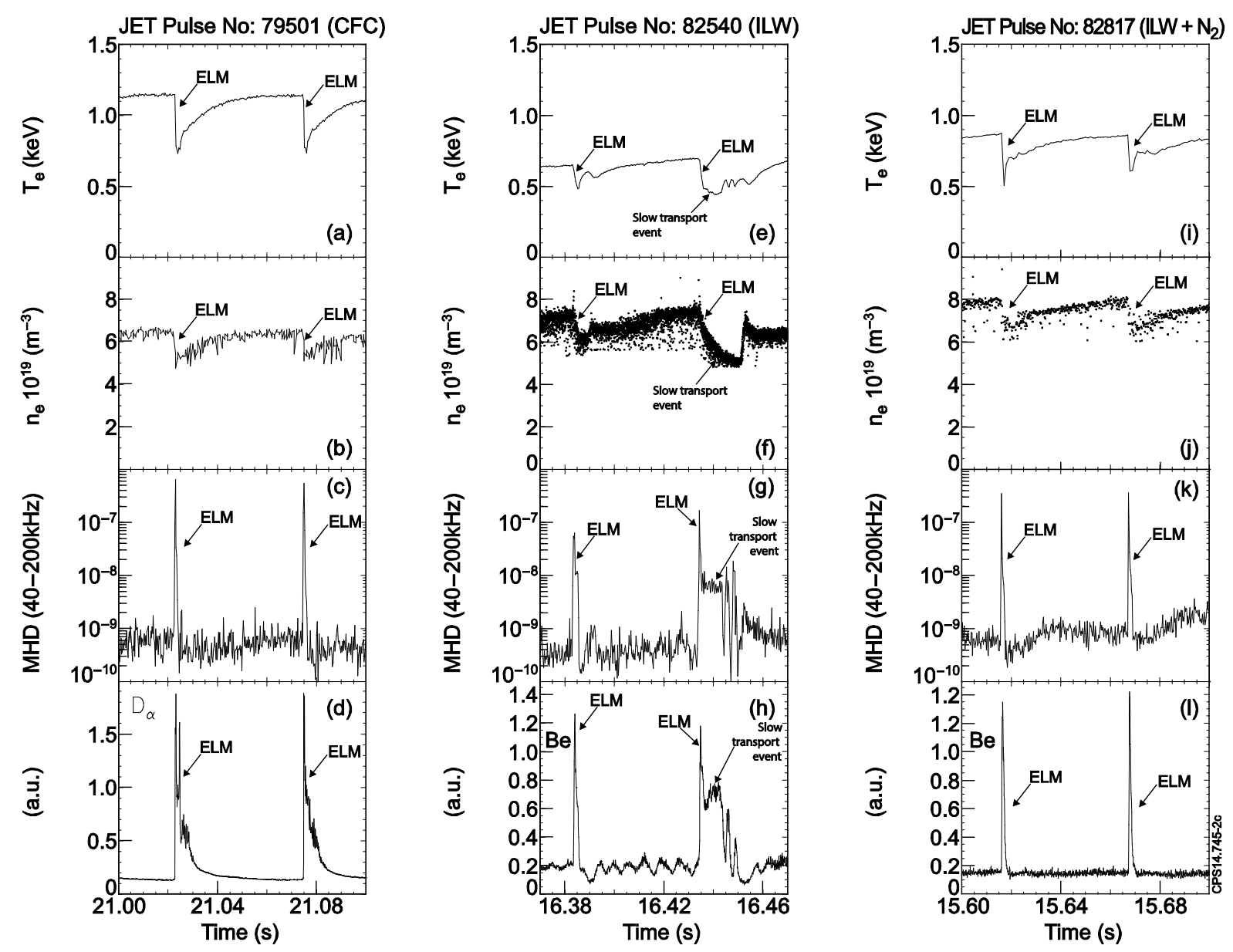

Figure 2. Time evolution at the pedestal top in a $0.1 \mathrm{~s}$ time window of electron temperature (first row), electron density (second row), high frequency MHD activity (third row) and $D_{\alpha}$ signal (fourth row) for a high- $\delta$ JET-C shot (first column), a high- $\delta$ JET-ILW without $N_{2}$ seeding (second column) and a high- $\delta$ JET-ILW with $N_{2}$ seeding (third column).

In this work, the collapses characterized by only ELMs are treated separately from those characterized by "ELMs"+"slow transport events". The time scale of the ELM collapse is further discussed in the remaining part of this Section. The ELM losses are described in Sections 4 and 5. The losses produced when the ELMs are followed by the slow transport events are discussed in Section 6.

To quantify the time scale of the ELM collapse, figure 4 shows the time evolution of the temperature and density at the top of the pedestal for all the ELMs within a steady phase of 
the shots discussed in figure 2. A steady phase has been defined as a time interval at least $1 \mathrm{~s}$ long with constant $\mathrm{H}_{98}, \beta_{\mathrm{N}}$, line averaged density and regular ELM frequency. The signals have been synchronized in order to have each ELM collapse at $\mathrm{t}=0$ and have been normalized to the pre-ELM value. For the JET-C shot, the time scale of the ELM collapse is $\tau_{\mathrm{ELM}}=0.6 \pm 0.1 \mathrm{~ms}$ for the pedestal temperature and $\tau_{\mathrm{ELM}}=0.4 \pm 0.3 \mathrm{~ms}$ for the pedestal density, see figures $4(\mathrm{a})$ and $4(\mathrm{~b})$.

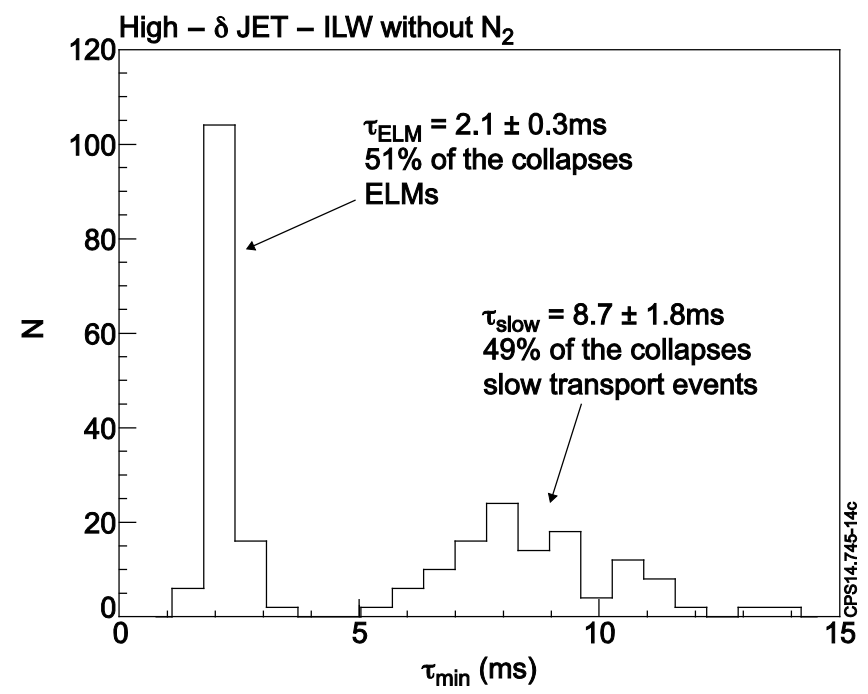

Figure 3. Distribution of the time to reach the minimum pedestal $T_{e}$ after the beginning of the collapse. The y-axis shows the number of collapses with a specific $\tau_{\min }$. All the collapses $(\approx 250)$ during a stationary phase in the high- $\delta$ JET-ILW shots with no N2 seeding of the present data set are considered. The data with $\tau_{\min }<4 m s$ correspond to the collapses due to the ELMs only and are used to identify the time scale of the ELM collapse $\tau_{E L M}$. The data with $\tau_{\min }>4 m s$ correspond to the collapses due to ELMs followed by slow transport events.

The non-seeded JET-ILW shot has a behaviour significantly different from the non-seeded JET-C shot, see figure 4(c) and 4(d). First of all, the presence of the slow transport event can be identified by a bifurcation in the temperature time trace. Note that the first phase of the temperature collapse is very similar for all the ELMs, till t-t $\mathrm{ELM}_{\mathrm{EL}} \approx 1.7 \mathrm{~ms}$. Then, the temperature starts to recover for approximately $40 \%$ of the ELMs, while a further collapse (even though with a slower decay rate) is present for the remaining $\approx 60 \%$ of the ELMs till $t-t_{\text {ELM }} \approx 10 \mathrm{~ms}$. The density signal is significantly noisier, figure 4(d), but a similar behaviour is present.

The time scale of the ELM collapse in the non-seeded JET-ILW shot ( $\tau_{\mathrm{ELM}} \approx 1.7 \mathrm{~ms}$ for the temperature and $2.5 \mathrm{~ms}$ for the density) is significantly longer than in the JET-C shot ( $\tau_{\mathrm{ELM}} \approx 0.6 \mathrm{~ms}$ and $0.4 \mathrm{~ms}$ ).

In terms of the time scale of the collapse, the $\mathrm{N}_{2}$ seeded JET-ILW shot shows a behaviour more similar to the JET-C than to the unseeded JET-ILW shot, see figures 4(e) and 4(f). First of all, slow transport events are not present. Second, the time scales of the ELM collapse are $0.8 \mathrm{~ms}$ for $\mathrm{T}_{\mathrm{e}}$ and $1.2 \mathrm{~ms}$ for $\mathrm{n}_{\mathrm{e}}$, close to the JET-C values. 

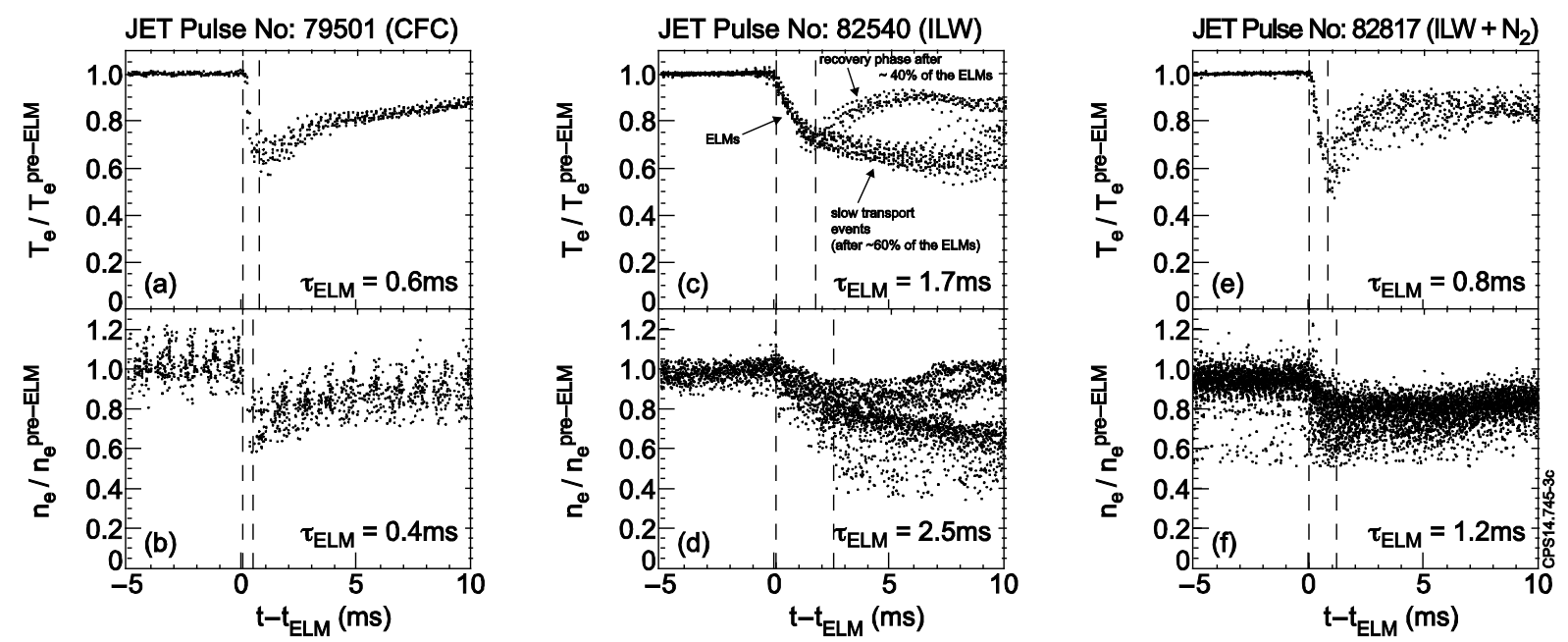

Figure 4. Time evolution ELM synchronized of electron temperature (first row) and electron density (second row) at the pedestal top for a high- $\delta$ JET-C shot (first column), high- $\delta$ JET$I L W$ with no $N_{2}$ seeding (second column) and high- $\delta$ JET-ILW with $N_{2}$ seeding (third column).
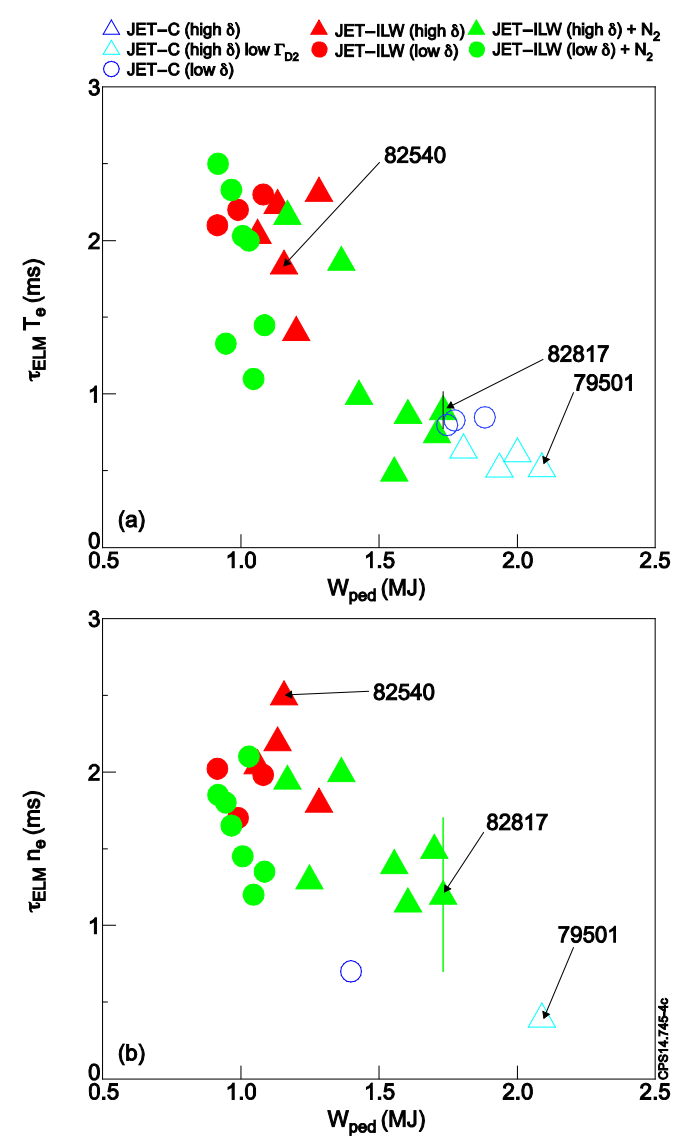

Figure 5. Time scale of the ELM collapse $\tau_{E L M}$ (no slow transport events included) versus the pedestal energy $W_{\text {ped }}$ for the temperature collapse (a) and for the density collapse (b). The time scales are calculated with the ECE radiometer and the reflectometer respectively. The pedestal energy is calculated using the HRTS as described in section 5. The typical error bars are in the range 0.1-0.4ms for the electron temperature and 0.4-0.8ms for the electron density (error bars are shown for the 82817 shot only). 
The time scale of the ELM collapse for the shots of the present data set is shown in figure 5 versus the pedestal stored energy. $\tau_{\mathrm{ELM}}$ is larger than $1.5 \mathrm{~ms}$ for the non-seeded JET-ILW plasmas (red full symbols) and lower than 1ms for the JET-C plasmas (blue empty symbols). The seeded high- $\delta$ JET-ILW plasmas can reach $\tau_{\text {ELM }}<1 \mathrm{~ms}$, a value comparable to the JET-C shots. For the low- $\delta$ JET-ILW, no clear correlation between $\tau_{\mathrm{ELM}}$ and the pedestal energy is observed. In fact, the $\tau_{\mathrm{ELM}}$ range is very large, from $1 \mathrm{~ms}$ to $2.5 \mathrm{~ms}$ while the pedestal energy is approximately constant ( $\mathrm{W}_{\text {ped }} \approx 1 \mathrm{MJ}$ ).

The data shown in figure 5 extend those of figure 4 to a larger data set and reinforce the result that $\tau_{\mathrm{ELM}}$ is higher in the non-seeded JET-ILW plasmas than in the JET-C plasmas and that JET-ILW can reach $\tau_{\text {ELM }}$ comparable to JET-C if $\mathrm{N}_{2}$ is seeded. A discussion on the correlation of $\tau_{\text {ELM }}$ with plasma parameters such as collisionality, $Z_{\text {eff }}$ and resistivity is presented in Section 7.
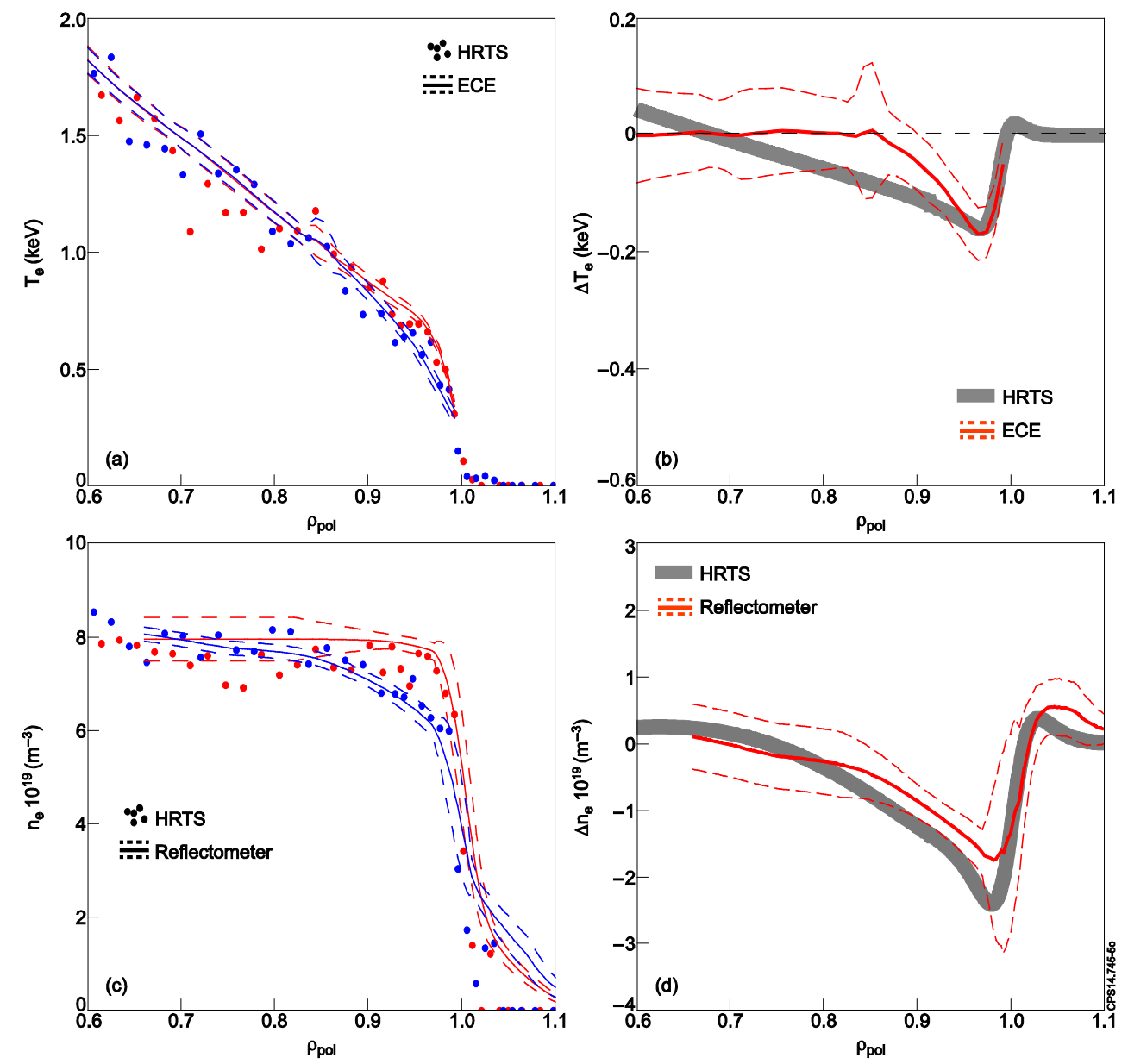

Figure 6. Profiles for the non-seeded JET-ILW plasma 82540. (a) Electron temperature profiles before (red) and after (blue) the ELM as measured by the HRTS (dots) and by the ECE radiometer. The continuous lines represent the average of the ECE profiles and the dashed lines represent the spread of the profiles. (b) Profile of the temperature collapse during the ELM as measured by the ECE (thin orange lines) and by the HRTS (thick grey line). The HRTS temperature collapse is calculated from the pre-and post-ELM fits obtained with a modified hyperbolic tangent function. Frame (c) shows the density profiles as measured by the reflectometer (lines) and the HRTS (dots) and frame (d) the profile of the density collapse (reflectormeter in thin orange lines and by the HRTS in the thick grey line). 


\section{PEDESTAL ELECTRON TEMPERATURE AND DENSITY COLLAPSE DURING ELMS}

This section quantifies the $\mathrm{T}_{\mathrm{e}}$ and $\mathrm{n}_{\mathrm{e}}$ drops due to ELMs. The drops due to the combined process "ELM"+"slow transport event" are discussed in section 6.

Figure 6 show the $T_{e}$ and $n_{e}$ profiles before the ELM and at the minimum of the ELM collapse for the non-seeded JET-ILW shot 82540 . The $T_{e}$ and $n_{e}$ profiles are determined from the ECE radiometer and the reflectometer respectively. Pre-ELM profiles have been calculated by averaging all the profiles in a time window from $-5 \mathrm{~ms}$ to $-1 \mathrm{~ms}$ before the ELMs. PostELMs profiles have been averaged in an approximately $0.1 \mathrm{~ms}$ long time window centred at the minimum of the signal after the ELM collapse. For comparison, figure 6 shows also the profiles from the HRTS. Due to the low sampling frequency $(20 \mathrm{~Hz})$, the HRTS cannot provide the profile evolution during a single ELM, so each measurement has been synchronized to its nearest ELM. The HRTS pre-ELM profiles have been obtained by considering the measurements in a time window from -10ms to -1ms before an ELM and the post-ELM profiles in a narrow time window after the ELM. The post-ELM time window is optimized to be as short as possible, but to include at least two profiles. It is typically not longer than $0.5 \mathrm{~ms}$.

Figure 6(a) shows the average temperature profiles and figure 6(b) the corresponding profile collapse due to the ELM. The pre-ELM and post-ELM density profiles and the corresponding profile collapse are shown in figures 6(c) and 6(d). The profiles from the ECE radiometer and from the reflectometer are in good with those from the HRTS.

\subsection{The $T_{\mathrm{e}}$ collapse due to ELMs}

The maximum $T_{e}$ collapse due to ELMs for the non-seeded JET-ILW plasma 82540 is $\Delta \mathrm{T}_{\mathrm{e}} \approx 150 \mathrm{eV}$, as shown in figure $6(\mathrm{~b})$. The results for the entire data set with the comparison between JET-C and JET-ILW are shown in figures 7(a) for the high- $\delta$ and in figure 7(b) for the low $-\delta . \Delta \mathrm{T}_{\mathrm{e}}$ is calculated at the radial position where the profile collapse is maximum.

The temperature collapse is significantly lower in the non-seeded JET-ILW plasmas than in the JET-C plasmas, both for the high- $\delta$ and the low- $\delta$.

When nitrogen is seeded into the high- $\delta$ JET-ILW plasmas, the $T_{e}$ drop can reach values comparable to the JET-C, both in terms of absolute drops, with $\Delta \mathrm{T}_{\mathrm{e}}$ up to $350 \mathrm{eV}$, and in terms of relative drops, with $\Delta \mathrm{T}_{\mathrm{e}} / \mathrm{T}_{\mathrm{e}}{ }^{\text {ped }}$ up to $40-50 \%$. When nitrogen is seeded into the low- $\delta$ JETILW plasmas, a marginal increase in the pedestal temperature is obtained, but $\Delta \mathrm{T}_{\mathrm{e}}$ remains significantly smaller than in the low- $\delta$ JET-C shots.

\subsection{The $n_{\mathrm{e}}$ collapse due to ELMs}

The maximum $n_{e}$ collapse due to ELMs for the non-seeded JET-ILW plasma 82540 is is $\Delta \mathrm{n}_{\mathrm{e}} \approx 2.0 \cdot 10^{19} \mathrm{~m}^{-3}$, as shown in figure 6(d). The electron density collapse for the entire data set is shown in figures 7(c) and 7(d) for high- $\delta$ and low- $\delta$ respectively.

In the high- $\delta$ shots, $\Delta \mathrm{n}_{\mathrm{e}}$ tends to be higher in the seeded JET-ILW plasmas than in the nonseeded JET-ILW, both in terms of absolute drops $\left(\Delta \mathrm{n}_{\mathrm{e}} \approx 1.4-2.5 \cdot 10^{19} \mathrm{~m}^{-3}\right.$ in the seeded and $\Delta \mathrm{n}_{\mathrm{e}} \approx 0.9-1.6 \cdot 10^{19} \mathrm{~m}^{-3}$ in the non-seeded $)$ and relative drops $\left(\Delta \mathrm{n}_{\mathrm{e}} / \mathrm{n}_{\mathrm{e}}{ }^{\text {ped }} \approx 15-25 \%\right.$ in the seeded and $\Delta \mathrm{n}_{\mathrm{e}} / \mathrm{n}_{\mathrm{e}}^{\text {ped }} \approx 12-20 \%$ in the seeded).

The density collapse of the high- $\delta$ JET-C plasmas depends on $\Gamma_{\mathrm{D} 2}$ level. The low gas shots [cyan triangles in figure $7(\mathrm{c})$ ] have $\mathrm{n}_{\mathrm{e}}{ }^{\text {ped }}$ and $\Delta \mathrm{n}_{\mathrm{e}}$ significantly lower than the shots (blue triangles) with $\Gamma_{\mathrm{D} 2}$ comparable to the JET-ILW. 
Considering only the shots with comparable gas level [red, green and blue symbols in figure 7(c)], the non-seeded JET-ILW plasmas have lower $\mathrm{n}_{\mathrm{e}}{ }^{\text {ped }}$ and $\Delta \mathrm{n}_{\mathrm{e}}$ then the JET-C, while the seeded JET-ILW has $\mathrm{n}_{\mathrm{e}}^{\text {ped }}$ and $\Delta \mathrm{n}_{\mathrm{e}}$ comparable to the JET-C.

Concerning the low- $\delta$ shots, no major difference is observed in the JET-ILW with and without $\mathrm{N}_{2}$ seeding. The JET-C plasmas have $\mathrm{n}_{\mathrm{e}}^{\text {ped }}$ and $\Delta \mathrm{n}_{\mathrm{e}}$ lower than the JET-ILW plasmas.
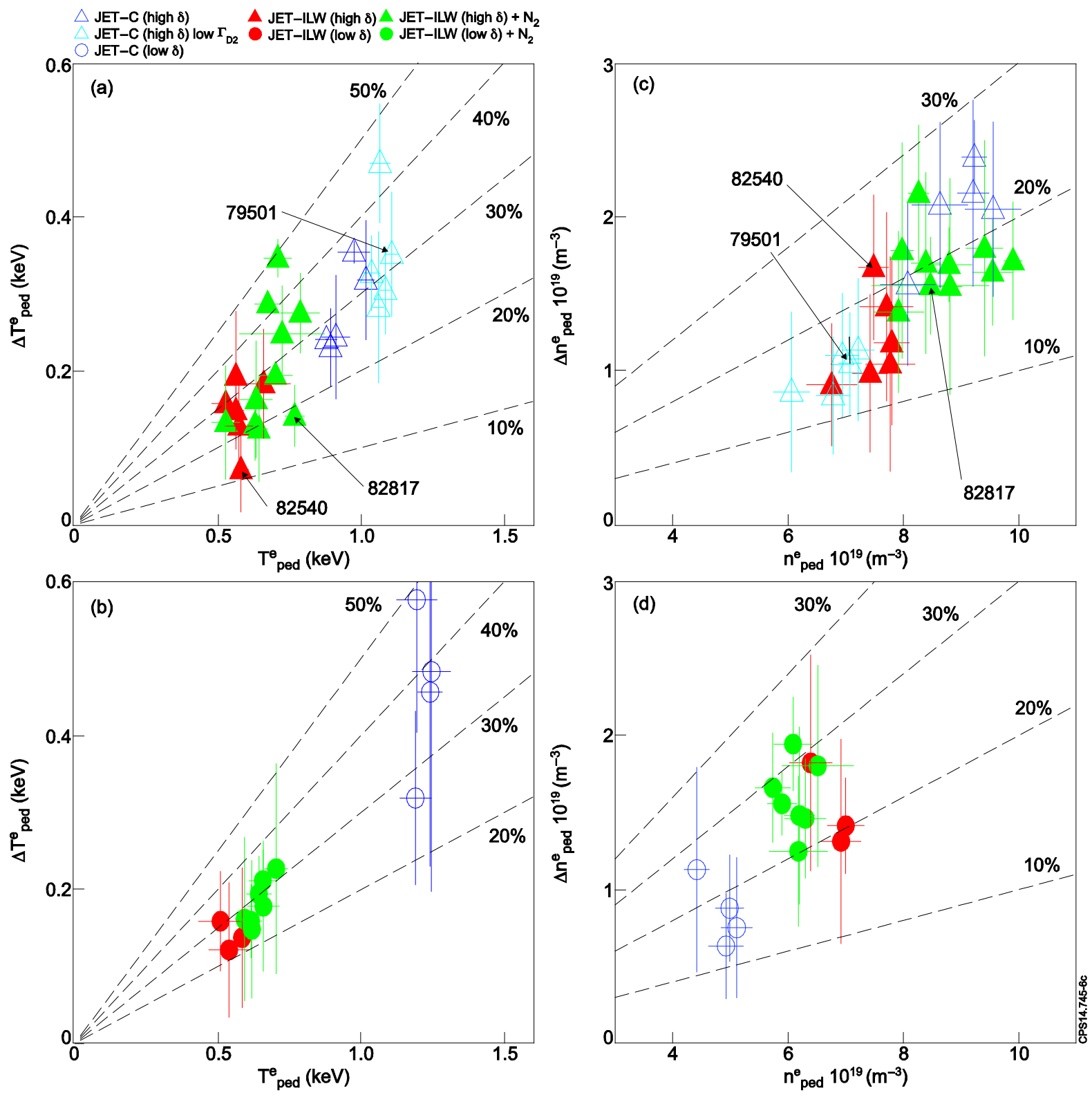

Figure 7. Electron temperature drop at the pedestal during ELMs versus the pedestal temperature for high and low triangularity plasmas, frames (a) and (b) respectively. Electron density drop at the pedestal versus the pedestal density for high and low triangularity plasmas, frames (c) and (d) respectively. The dashed lines show constant ratios between the pedestal drop and the pedestal value. 


\section{ELM ENERGY LOSSES}

The electron thermal energy is calculated by volume integrating the electron temperature and density profiles:

$$
W_{t h}^{e}=\frac{3}{2} k \int T_{e} n_{e} d V
$$

The electron ELM energy losses are calculated as the $\mathrm{W}^{\mathrm{e}}$ th difference between the pre- and the post-ELM phase:

$$
\begin{aligned}
\Delta W_{E L M}^{e} & =\frac{3}{2} k\left[\int T_{e} n_{e} d V-\int\left(T_{e}-\Delta T_{e}\right)\left(n_{e}-\Delta n_{e}\right) d V\right] \approx \\
& \approx \frac{3}{2} k\left[\int \Delta T_{e} n_{e} d V+\int T_{e} \Delta n_{e} d V\right]=\Delta W_{c o n d}^{e}+\Delta W_{c o n v}^{e}
\end{aligned}
$$

where $\Delta \mathrm{T}_{\mathrm{e}}$ and $\Delta \mathrm{n}_{\mathrm{e}}$ are the temperature and density collapses during the ELM, and $\Delta \mathrm{W}^{\mathrm{e}}$ cond, $\Delta \mathrm{W}^{\mathrm{e}}$ conv represent the conductive and convective losses respectively. The cross-term in equation 2 is negligible.

The total electron ELM energy losses versus the pedestal stored energy $W_{\text {ped }}$ is shown in figure 8(a). The pedestal stored energy $\mathrm{W}_{\text {ped }}$ is calculated by considering both the electron pedestal pressure and the ion pedestal pressure using the HRTS for the electron and the charge exchange for the ions as described in [13]. For the JET-ILW plasmas $T_{i}=T_{e}$ is assumed.

$\Delta \mathrm{W}^{\mathrm{e}}$ ELM is in the range $100-220 \mathrm{~kJ}$ for the JET-C plasmas, significantly larger than the nonseeded ILW plasmas that are characterized by $\Delta \mathrm{W}^{\mathrm{e}}{ }_{\mathrm{ELM}} \approx 30-70 \mathrm{~kJ}$. The ELM energy losses for the JET-C plasmas are larger also in terms of the relative drop, with $\Delta \mathrm{W}^{\mathrm{e}}$ ELM $/ \mathrm{W}_{\text {ped }} \approx 6 \%$ $10 \%$, for the JET-C and $\Delta \mathrm{W}_{\mathrm{ELM}}^{\mathrm{e}} / \mathrm{W}_{\text {ped }} \approx 4 \%-6 \%$ for the JET-ILW.

JET-ILW plasmas can reach energy losses comparable to the JET-C when nitrogen is seeded. In this case, the JET-ILW have losses in the range $\Delta \mathrm{W}_{\mathrm{ELM}}^{\mathrm{e}} \approx 40-130 \mathrm{~kJ}$ and $\Delta \mathrm{W}^{\mathrm{e}}{ }_{\mathrm{ELM}} / \mathrm{W}_{\text {ped }}$ up to $8 \%$. Note that the JET-ILW shots with losses comparable to the JET-C are the plasmas which reach a $\mathrm{W}_{\text {ped }}$ comparable to the JET-C plasmas.

The contributions of the convective and conductive terms to the total ELM energy losses are shown in figure 8(b) and 8(c) respectively. The difference in the convective losses between JET-C and JET-ILW plasmas is evident mainly for the high- $\delta$ shots, with $\Delta \mathrm{W}^{\mathrm{e}}$ conv often larger than $50 \mathrm{~kJ}$ in the JET-C and $\Delta \mathrm{W}_{\text {conv }}^{\mathrm{e}}<50 \mathrm{~kJ}$ in the non-seeded JET-ILW. The difference is even more evident in the conductive losses with $\Delta \mathrm{W}^{\mathrm{e}}$ cond $70-150 \mathrm{~kJ}$ for the JET-C and $\Delta \mathrm{W}^{\mathrm{e}}$ cond $20-50 \mathrm{~kJ}$ for the JET-ILW. The ratio between the conductive losses and the pedestal energy increases from $2 \%-4 \%$ for the non-seeded JET-ILW plasmas to $4 \%-8 \%$ for the JET-C plasmas. The seeded JET-ILW plasmas with $\mathrm{W}_{\text {ped }}$ comparable to the JET-C plasmas have also comparable conductive losses. 

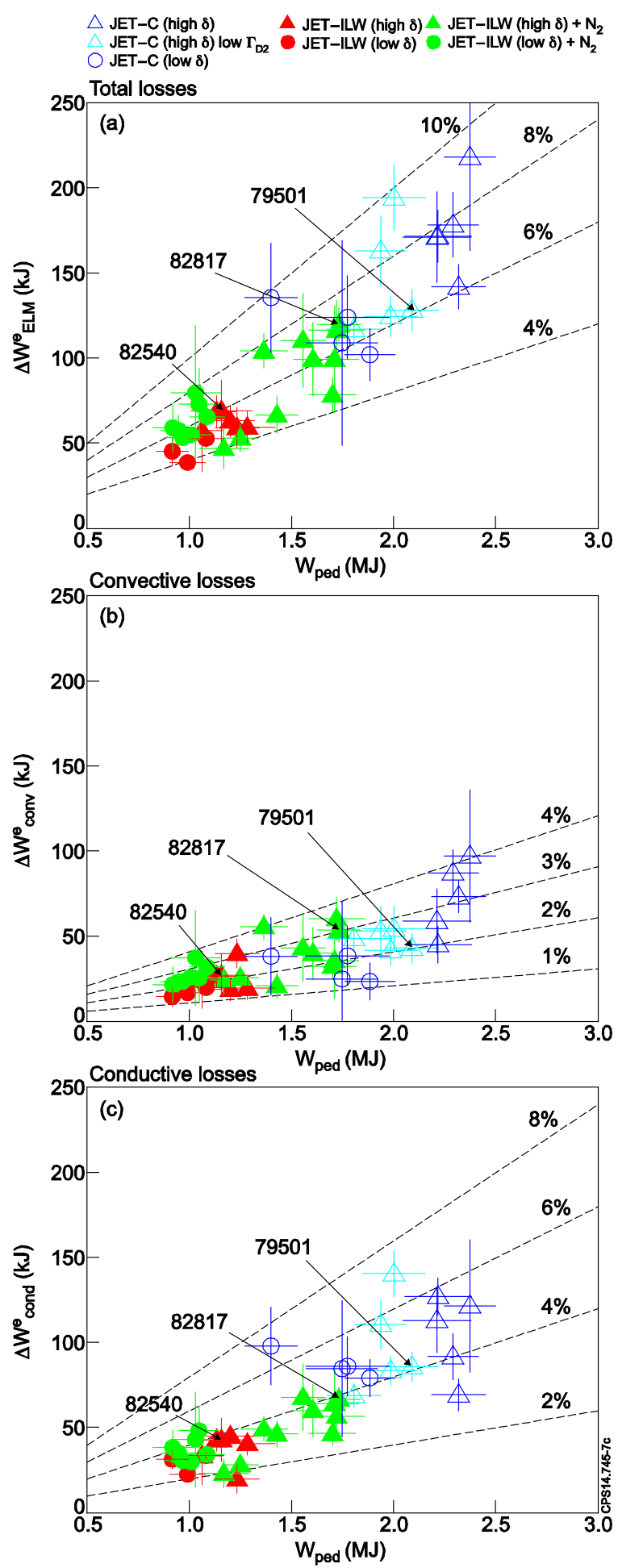

Figure 8. Electron ELM energy losses versus pedestal stored energy for the total losses (a), the convective losses (b) and the conductive losses (c). The dashed lines highlight constant ratios of the ELM losses versus the pedestal stored energy.

Note that the ELM energy losses depend not only on the drops at the pedestal but also on the width of the ELM affected area. The ELM affected volume for the temperature seems marginally larger in the JET-C plasmas, with a radial width from $\rho_{\theta} \approx 0.8$ to $\rho_{\theta} \approx 1.0$ than in the non-seeded ILW plasmas, which have a radial width $\rho_{\theta} \approx 0.85-1.0$. No significant difference is observed in the ELM affected volume for the electron density. However, due to the large uncertainty in determining the width, conclusive claims are not possible. 


\section{THE SLOW TRANSPORT EVENT IN JET-ILW PLASMAS}

An ELM in the JET-ILW plasma is sometimes followed by a further transport event that occurs on a longer time scale. The typical temporal behaviour of the slow transport events is shown in figure 3(c). After an initial phase that is similar for all the ELMs (from $t$ - $_{\text {ELM }}=0 \mathrm{~ms}$ to $\mathrm{t}-\mathrm{t}_{\mathrm{ELM}} \approx 1.7 \mathrm{~ms}$ ), the temperature starts to recover for $\approx 40 \%$ of the ELMs while it continues to decrease till $\mathrm{t}-\mathrm{t}_{\mathrm{ELM}} \approx 10 \mathrm{~ms}$ for the remaining $60 \%$ of the ELMs. The rate of reduction of the temperature is clearly lower during the slow transport events. The electron density has a relatively similar behaviour, as shown in figure 3(d).

The slow transport events can be relatively frequent in a JET-ILW plasma. Figure 9 shows the frequency of the slow transport events for all the shots in the data set versus the pedestal stored energy. The frequency has been calculated for each shot as the number of the slow transport events, $\mathrm{N}_{\text {slow }}$, divided by the total number of ELM collapses, $\mathrm{N}_{\text {tot }}$ (including both the standard ELMs and the ELMs followed by the slow transport event). The slow transport events can be present in up to 70\% of the ELMs. However, the seeded JET-ILW plasmas with $\mathrm{W}_{\text {ped }}>1.5 \mathrm{MJ}$ have no slow transport events. In JET-C, no ELM is followed by a slow transport event. The absence of slow transport events in JET-ILW plasmas with high $\mathrm{W}_{\text {ped }}$ has been investigated also in a wider data set. To date, the number of JET-ILW shots with high $\mathrm{W}_{\text {ped }}$ is limited, so conclusive claims are not possible, but no slow transport events at high $\mathrm{W}_{\text {ped }}$ have been observed so far.

Apart from the fact that slow transport events are present only in plasmas with $\mathrm{W}_{\text {ped }}<1.5 \mathrm{MJ}$, no strong correlation is observed between the fraction of the slow transport events and the pedestal stored energy. At the moment, the origin of the slow transport event is unclear.

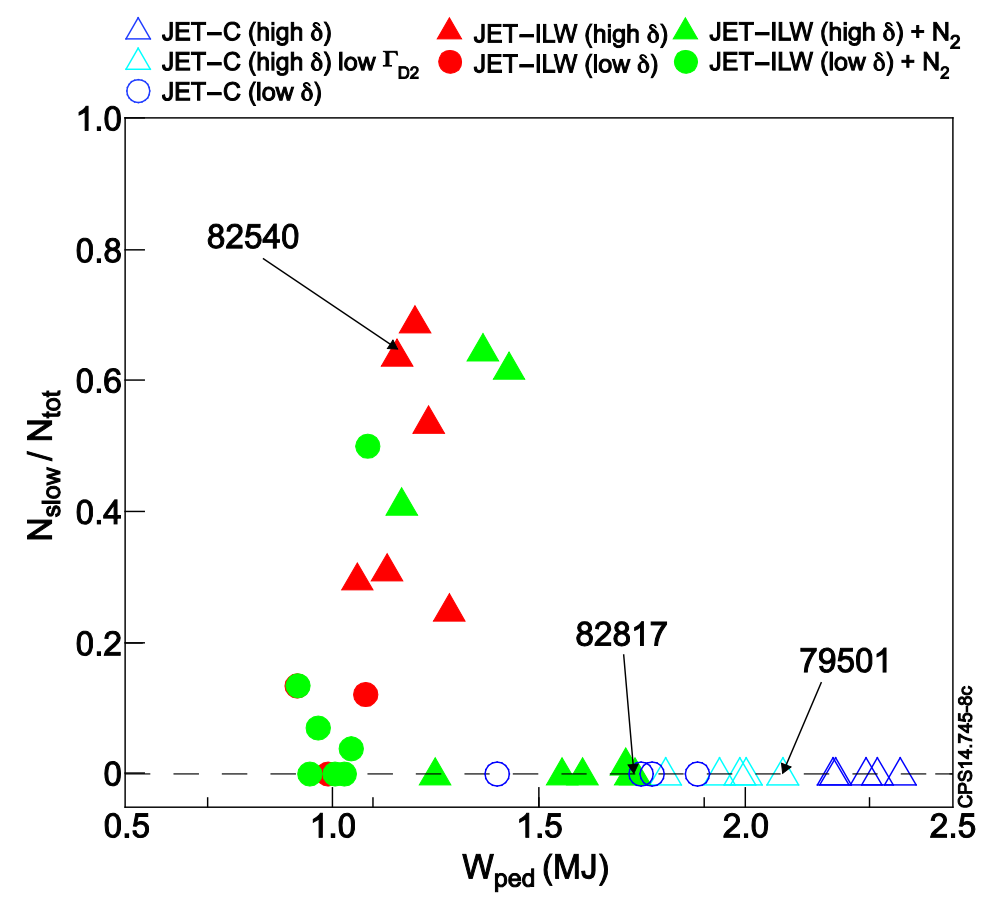

Figure 9. Ratio between the number of slow transport events and the total number of ELMs (including both standard ELMs and those followed by the slow transport event) versus the pedestal energy.

The net power across the separatrix $\left(\mathrm{P}_{\text {sep }}\right)$ is above the $\mathrm{L}-\mathrm{H}$ power threshold. $\mathrm{P}_{\mathrm{L}-\mathrm{H}}$, as determined using the Martin scaling law [28], is in the range 9-12MW and $\mathrm{P}_{\mathrm{sep}} / \mathrm{P}_{\mathrm{L}-\mathrm{H}} \approx 1.1-1.5$ for the present set of JET-ILW plasmas. So, the slow transport event is likely not a full back transition to the L-mode. Moreover, the pedestal structure is observed also at the maximum of 
the collapse both in the temperature and in the density profiles. This is shown in figure 10, where the profiles before the ELM (red symbols) and at the maximum of the collapse during the slow transport events (blue symbols) are shown.
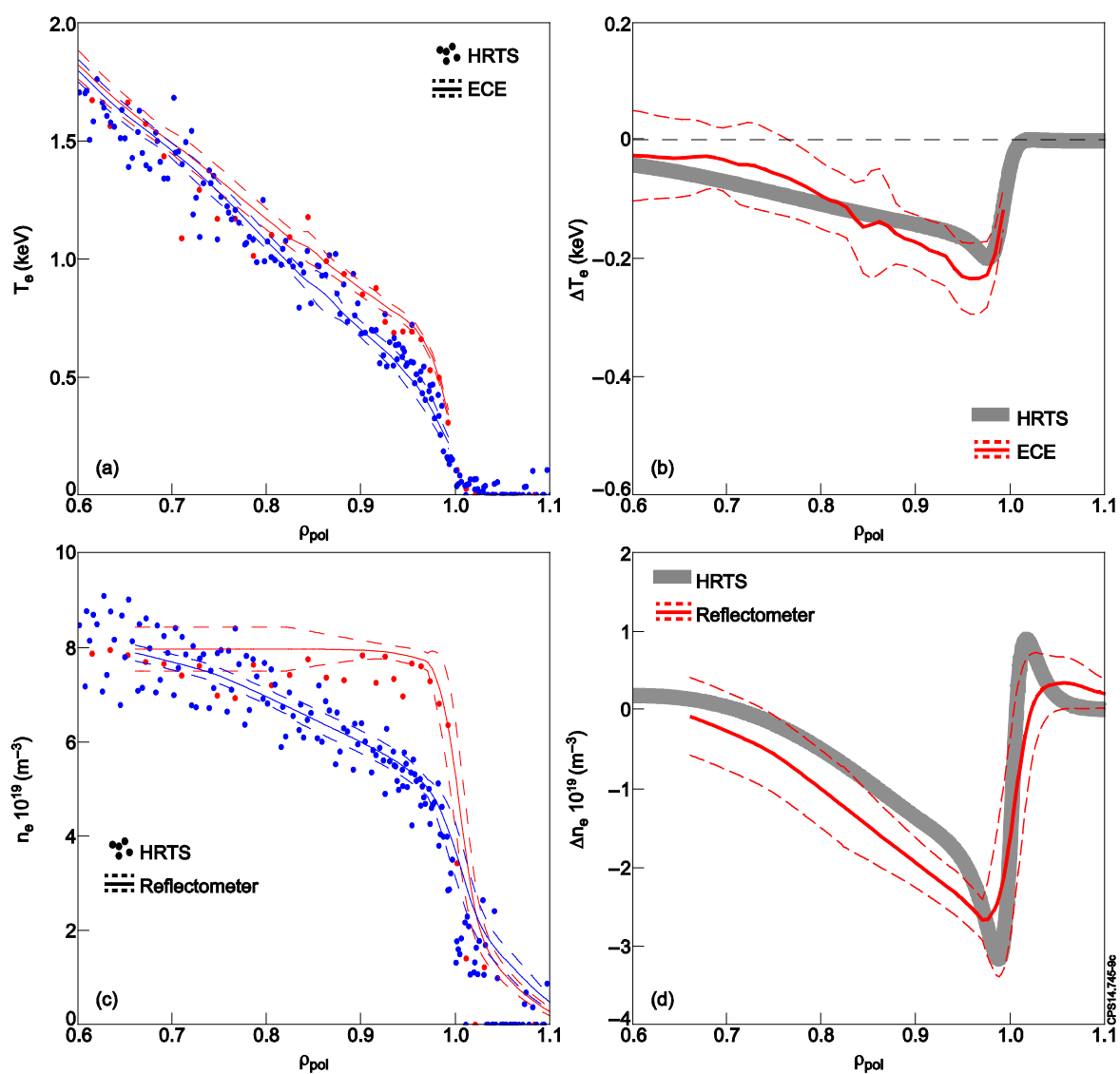

Figure 10. Profiles in the pre-ELM phase and after the slow transport event for the nonseeded JET-ILW plasma 82540. Pre-ELMs profiles, red data in frames (a) and (c), are calculated in time window from -5ms to -1ms before the ELMs. Post-ELMs profiles, blue data in frames (b) and (d), have been calculated in an approximately 0.5ms long time window centered at the minimum of the signal after the beginning of the slow transport event. The profiles of the temperature and density collapses are shown in frame (b) and (d) respectively.

The temperature and density collapse during the slow transport events for shot 82540 are shown in figures 10(c) and 10(d) respectively. The collapse is calculated as the drop of the profile from before the beginning of the ELM till the minimum value reached during the slow transport event. The collapse is therefore representative of the entire process "ELM"+"slow transport event". The drops are $\Delta \mathrm{T}_{\mathrm{e}} \approx 200 \mathrm{eV}$ and $\Delta \mathrm{n}_{\mathrm{e}} \approx 3 \cdot 10^{19} \mathrm{~m}^{-3}$, both larger than the drops during the standard ELMs, which are $\Delta \mathrm{T}_{\mathrm{e}} \approx 150 \mathrm{eV}$ and $\Delta \mathrm{n}_{\mathrm{e}} \approx 2 \cdot 10^{19} \mathrm{~m}^{-3}$ (figures $6 \mathrm{~b}$ and $6 \mathrm{~d}$ ).

These results are summarized in figure 11 , where in frame (a) and (b) the temperature and density drops at the pedestal are shown versus the pedestal temperature and density. For reference, the temperature and density collapses during ELMs are shown as well. The density drops due to the process "ELM"+"slow transport event" (black squares) are in the range $\Delta \mathrm{n}_{\mathrm{e}} / \mathrm{n}_{\mathrm{e}}{ }^{\text {ped }} \approx 30 \%-50 \%$, significantly larger than those due to ELMs, which are in the range $\Delta \mathrm{n}_{\mathrm{e}} / \mathrm{n}_{\mathrm{e}}^{\text {ped }} \approx 10-25 \%$.

The slow transport event increases further the collapse of $\mathrm{T}_{\mathrm{e}}{ }^{\text {ped }}$ and $\mathrm{n}_{\mathrm{e}}{ }^{\text {ped }}$ due to the ELM. Therefore, the total energy losses of the plasma when the ELM is followed by a slow transport event are larger than the losses produced by the ELM alone. The electron energy losses are shown in figure 11(c). The energy losses due to the process "ELM"+"slow transport event" are shown with black squares. The energy losses due to the process "ELM"+"slow transport 
event" can reach up to $10 \%$ of the pedestal stored energy and are significantly larger than the ELM losses of the JET-ILW plasmas.
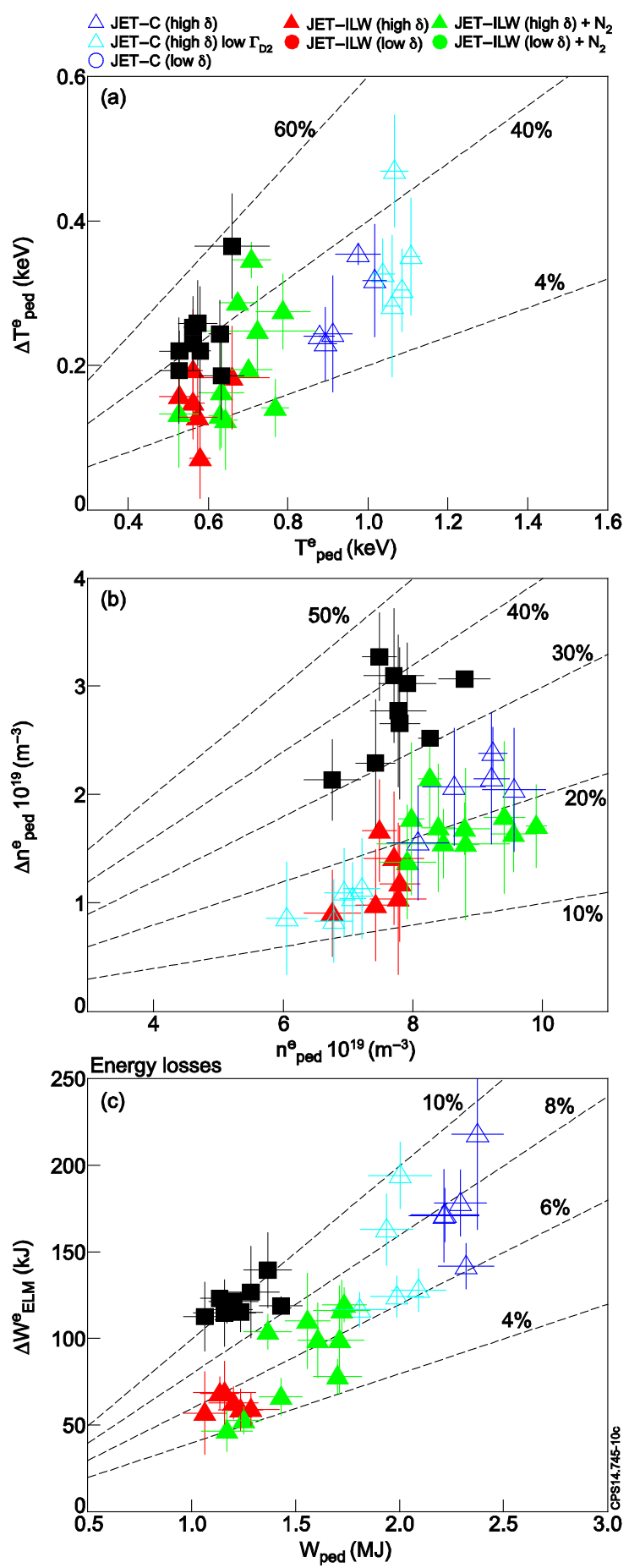

Figure 11. Pedestal drops for electron temperature (a) and density (b) versus the pre-ELM pedestal value. Electron energy losses versus the pedestal energy (c). Black squares highlight the drops due to the slow transport events. Coloured symbols highlight the drops due to the ELMs. 


\section{DISCUSSION.}

As discussed in Section 3 and in Section 6, the ELMs in the JET-ILW are sometimes followed by a second collapse, called slow transport event, that increases the energy losses. Section 5 shows that the ELM energy losses (i.e. no slow transport events included) are lower in the non-seeded JET-ILW than in the JET-C, while the $\mathrm{N}_{2}$ seeded JET-ILW can have ELM energy losses comparable to the JET-C. The $\mathrm{N}_{2}$ seeded JET-ILW can reach also ELM time scales comparable to the JET-C.

The ELM energy losses seem well correlated with the pedestal stored energy, while the time scale of the ELM collapse, in particular for the low- $\delta$ JET-ILW, does not seem to be strongly correlated with $\mathrm{W}_{\text {ped. }}$. This section investigates possible parameters that might regulate the behaviour of the ELM energy losses and the ELM time scale.

Earlier works [4, 29, 30, 31] have shown that the relative ELM energy losses scale with the pedestal collisionality $v_{\mathrm{e}}{ }^{*}($ neo $)$. The collisionality is defined as $v_{\mathrm{e}}{ }^{*}($ neo $)=\mathrm{qR}^{5 / 2} \mathrm{a}^{-3 / 2}\left(\lambda_{\text {ee }}\right)^{-1}$ where $\lambda_{\text {ee }}=1.7 \times 10^{17} \mathrm{~T}_{\mathrm{e}}^{2}(\mathrm{eV}) /\left[\mathrm{n}_{\mathrm{e}}\left(\mathrm{m}^{-3}\right) \ln \Lambda\right]$ is the electron-electron Coulomb collision mean free path, with the temperature and density calculated at the pedestal [4].

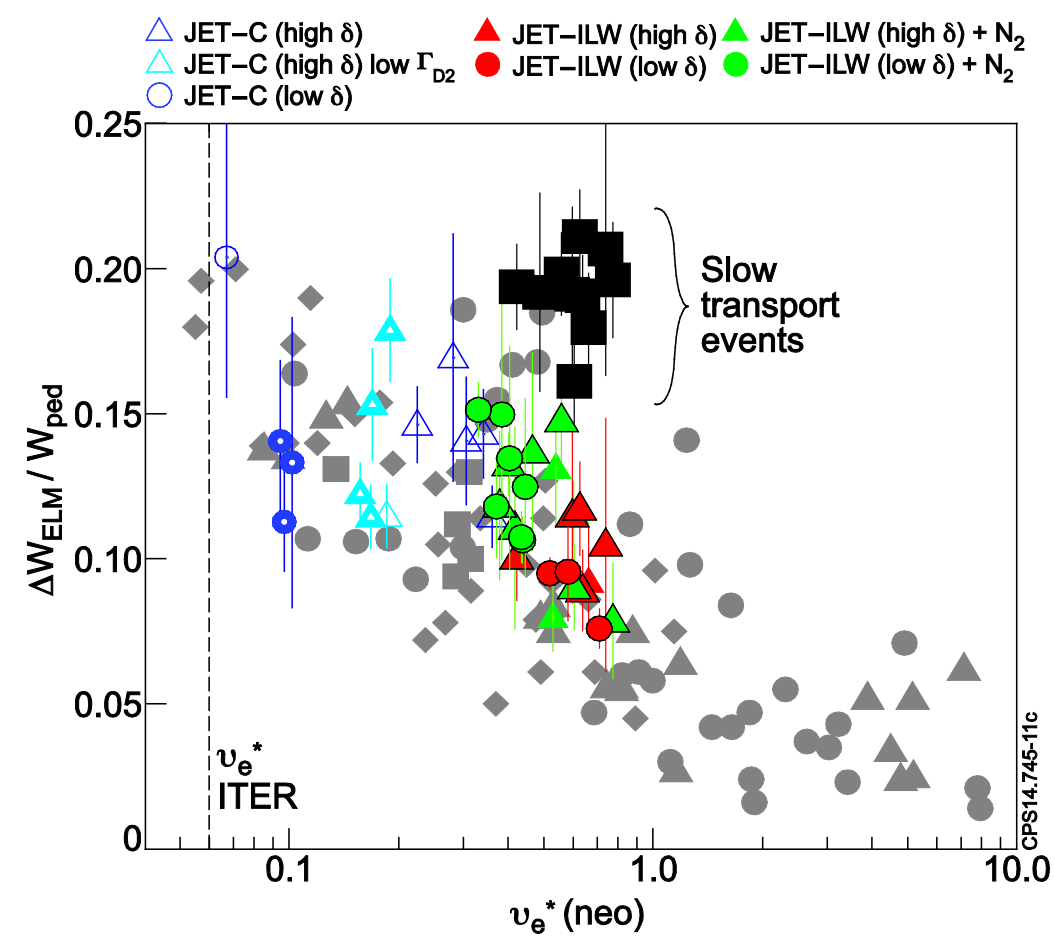

Figure 12. Relative ELM energy losses versus the pedestal collisionality for the present sets of JET-C data (blue, cyan, read and green symbols). The black squares highlight the energy losses during the slow transport events in the JET-ILW plasma. The grey data show the multimachine results described in [4]. The open symbols with thick borders and the full symbols with black borders highlight the data used in figure 13.

The present results and those described in [4] are compared in figure 12, where the total ELM energy losses $\Delta \mathrm{W}_{\text {ELM }}$ relative to the total pedestal stored energy $\mathrm{W}_{\text {ped }}$ versus the pedestal collisionality are shown. For the present data set, the total ELM energy losses $\Delta \mathrm{W}_{\text {ELM }}$ have been calculated using the electron ELM energy losses $\Delta \mathrm{W}_{\text {ELM }}^{\mathrm{e}}$, assuming $\mathrm{T}_{\mathrm{e}}=\mathrm{T}_{\mathrm{i}}$ and calculating the ion density from the line integrated effective charge using as main impurity carbon for the JET-C plasmas $\left(n_{i}=n_{e}\left(7-Z_{\text {eff }}\right) / 6\right.$ ), beryllium for the non-seeded JET-ILW plasmas $\left(n_{i}=n_{e}\left(5-Z_{e f f}\right) / 4\right)$ and nitrogen the seeded JET-ILW plasmas $\left(n_{i}=n_{e}\left(8-Z_{e f f}\right) / 7\right)$. The present data set follows reasonably well the earlier trend of the relative ELM energy losses versus the pedestal collisionality. In particular, the JET-ILW plasmas have energy losses comparable with the JET-C when their pedestal collisionality is reduced to the JET-C values. 
For comparison, the energy losses due to the process "ELM"+"slow transport event" are shown in figure 12 as black squares. These losses do not fit into the trend of $\Delta \mathrm{W}_{\text {ELM }} / \mathrm{W}_{\text {ped }}$ versus $v_{\mathrm{e}}{ }^{*}(\mathrm{neo})$ and are significantly larger than the JET-ILW energy losses due to the ELMs alone.

The pedestal collisionality is well correlated also the time scale of the ELM collapse, as shown in figure 13. A positive trend is present for both the high- $\delta$ and low $\delta$ plasmas. The ELM time scale is determined using the ECE radiometer (when available), so the data of figure 13 are a subset of the data of figure 12 .
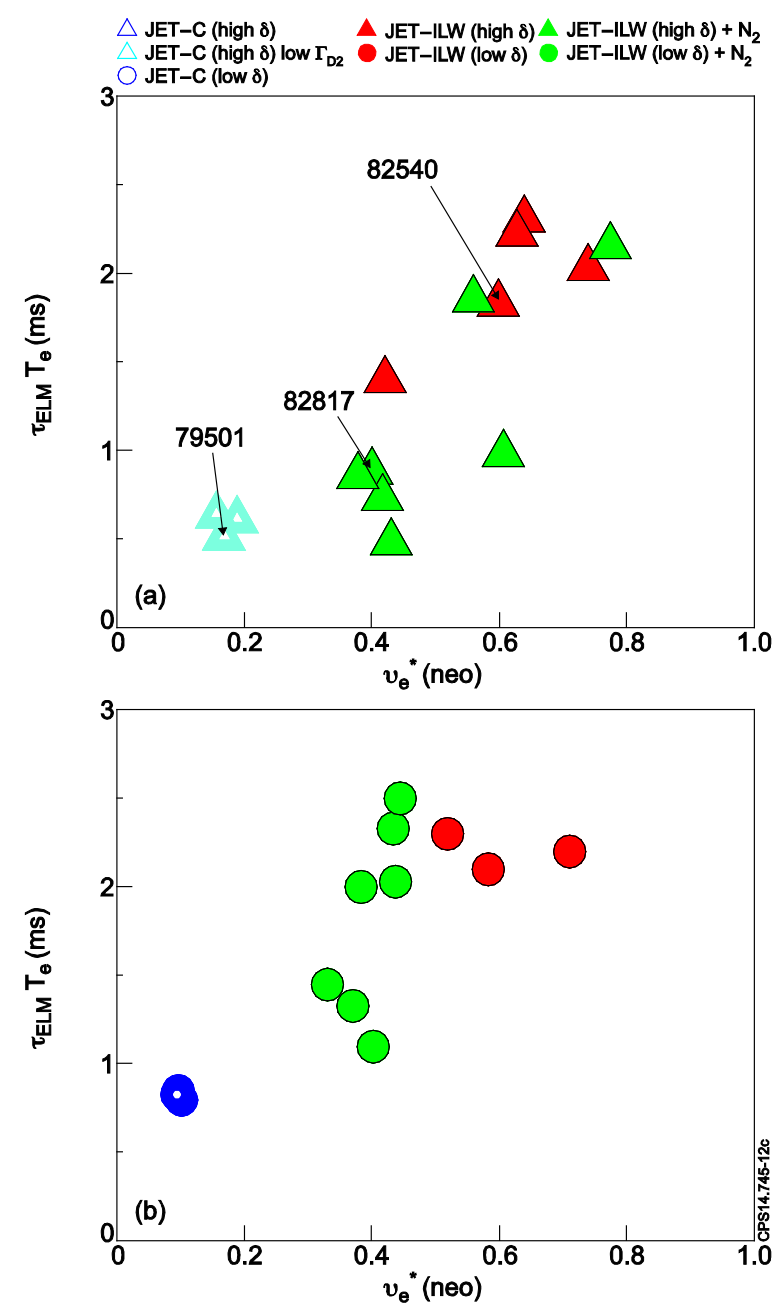

Figure 13. Temperature ELM time scale versus pedestal collisionality for the high- $\delta$ plasma (a) and the low- $\delta$ plasmas (b).

The confinement improvement with nitrogen seeding has been observed also in ASDEX Upgrade where it is associated with the correlation between $\mathrm{H}_{98}$ and the effective charge $\mathrm{Z}_{\text {eff }}$ [Schweinzer NF2011]. So far, in JET-ILW, a strong correlation between confinement and $\mathrm{Z}_{\text {eff }}$ has not been observed yet [9]. Nonetheless, the increase of the $\mathrm{N}_{2}$ seeding rate produces an increase in $\mathrm{Z}_{\mathrm{eff}}$ [9]. The N2 seeded JET-ILW plasmas analysed in this work have a seeding rate in the range $\Gamma_{\mathrm{N} 2}=0.5-3 \cdot 10^{22}(\mathrm{e} / \mathrm{s})$. The seeded shots with low $\Gamma_{\mathrm{N} 2}$ have $\mathrm{Z}_{\text {eff }}$ comparable to the non-seeded JET-ILW plasmas $\left(\mathrm{Z}_{\mathrm{eff}} \approx 1.3-1.4\right)$, while with high $\Gamma_{\mathrm{N} 2} \mathrm{Z}_{\mathrm{eff}} \approx 1.6$ which is comparable to the JET-C plasmas [9].

To investigate if the ELM properties are related to the change in Zeff, the first column figure 14 shows the time scale of the ELM collapse, the ELM energy losses and the fraction of slow transport events versus $Z_{\text {eff. }}$ The ELM time scale for the JET-ILW plasmas seems indeed well 
correlated with the effective charge. The seeded JET-ILW shots with $\mathrm{Z}_{\text {eff }}>1.5$ have a time scale comparable to the JET-C shots. On the other hand, there is no correlation for the JET-C plasmas, for which the ELM time scale is relatively constant but $\mathrm{Z}_{\text {eff }}$ varies from 1.6 to 2.2. In this sense, the ELM time scale is better correlated with the pedestal. However, we cannot exclude that the effective charge is related to the ELM time scale via other plasma parameters, such as the resistivity. The resistivity depends on both $Z_{\text {eff }}$ and $\mathrm{T}_{\mathrm{e}}{ }^{\text {ped }},\left[\eta=1.6 \cdot 10^{-9} \ln \Lambda \mathrm{Z}_{\text {eff }} /\right.$ $\left(\mathrm{T}_{\mathrm{e}}^{\mathrm{ped}}\right)^{3 / 2}$. Most of the variation of $\mathrm{Z}_{\text {eff }}$ in the present data set is obtained by seeding $\mathrm{N}_{2}$, which often leads to higher $\mathrm{T}_{\mathrm{e}}{ }^{\text {ped }}$. Since these effects may partially cancel out in the resistivity, figure 14(b) shows the ELM time scale versus $T_{e}{ }^{\text {ped }}$ and Figure 14(c) shows the ELM time scale versus the resistivity. The ELM time scales in the JET-ILW show a correlation with both $\mathrm{T}_{\mathrm{e}}{ }^{\text {ped }}$ and $\eta$. But considering only the shots with $\tau_{\mathrm{ELM}}<1 \mathrm{~ms}$ no clear correlation is present. Concerning the ELM energy losses, a weak positive trend with $Z_{\text {eff }}$ and $T_{e}{ }^{\text {ped }}$ is present, figures 14(d) and 14(e). In particular, JET-C plasmas have large $Z_{\text {eff }}$ and large energy losses while non-seeded JET-ILW shots have smaller $Z_{\text {eff }}$ and smaller energy losses. However, the seeded JET-ILW plasmas show no clear correlation between energy losses and $Z_{\text {eff. The }}$ correlation between the energy losses and the plasma resistivity is not strong either, as shown in figure 14(f).

As a final comment, even if the the slow transport events are present only in the JET-ILW plasmas, no clear correlation is found with $\mathrm{Z}_{\text {eff }}$, with $\mathrm{T}_{\mathrm{e}}{ }^{\text {ped }}$ or with the resistivity. This is shown in figures 14(g), 14(h) and 14(i).

The slow transport event in the JET-ILW has several similarities with what observed in ASDEX Upgrade and labelled phase "1b" of an ELM. As described in [18], the ELMs in ASDEX Upgrade are characterized by an initial phase ("1a") plus a second phase (" $1 b$ ") which produces a further collapse of $\mathrm{T}_{\mathrm{e}}$ and $\mathrm{n}_{\mathrm{e}}$. The " $1 \mathrm{~b}$ " phase is characterized by a density collapse that resembles the one observed in the slow transport events and shown in figure 10 of this paper. The "1b" ELM phase in ASEDX Upgrade is also characterized by a high frequency MHD activity that is lower than the "1a" ELM phase but higher than the pre-ELM phase, similar to what observed in figure 2(g) of the present paper. Moreover, the " $1 \mathrm{~b}$ " phase disappears when $\mathrm{N}_{2}$ is seeded. Finally, the disappearance of the " $1 \mathrm{~b}$ " phase in ASDEX Upgrade is not gradual, but more similar to a threshold effect, as discussed for the JET-ILW in Section 6. These observations suggest that the slow transport event in JET-ILW could be similar to the " $1 b$ " phase observed in ASDEX Upgrade ELMs. The origin of these events has not been identified yet. 

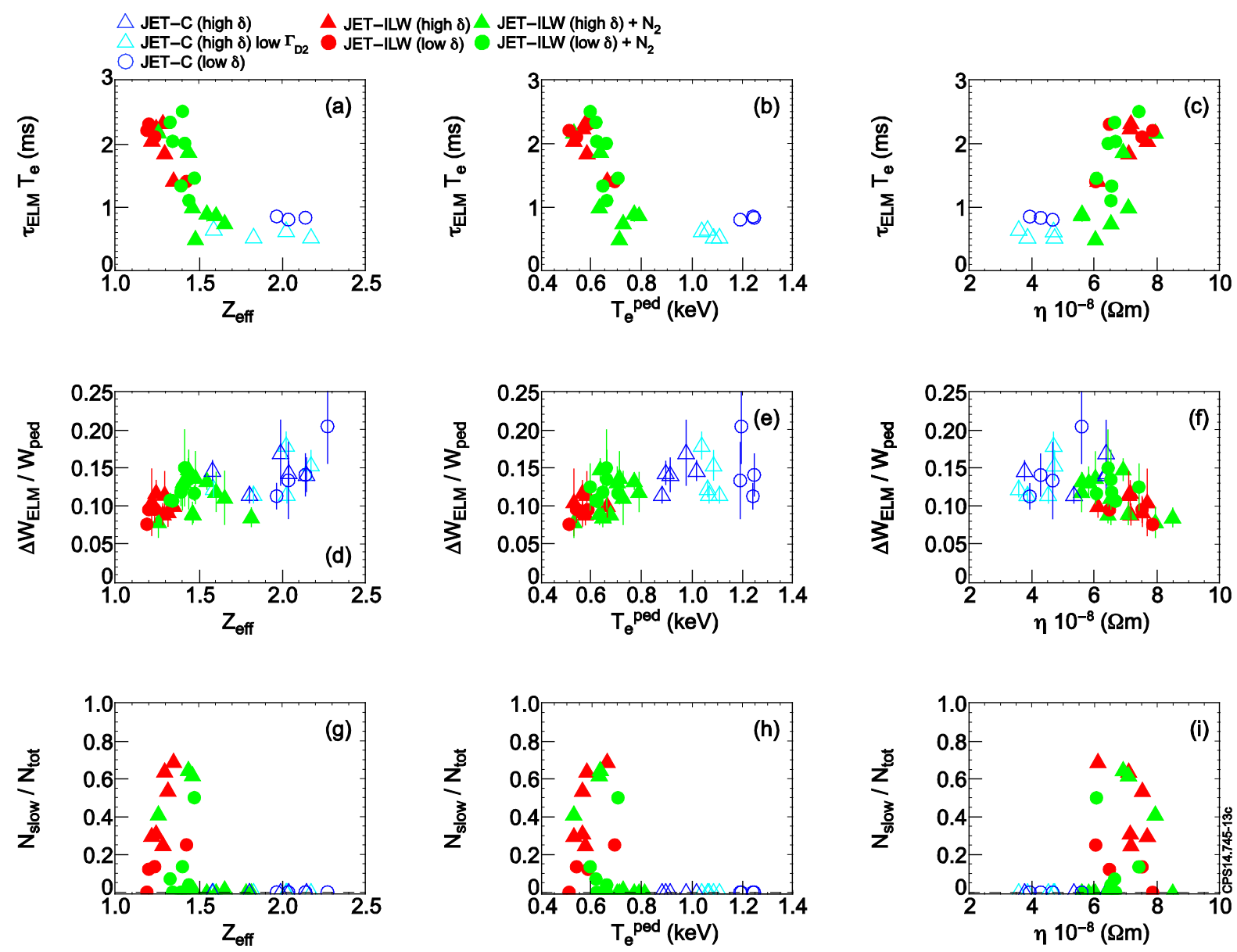

Figure 14. ELM time scale (a), (b) and (c), ELM energy losses (d), (e) and ( $f$ ), fraction of slow transport events (g), (h) and (i) versus the effective charge (first column), pedestal temperature (second column) and the plasma resistivity (third column).

\section{CONCLUSION.}

This work describes the $\mathrm{N}_{2}$ seeding effect on the time scale of the ELM collapse and on the ELM energy losses in JET-ILW and compares the results with similar non-seeded JET-C plasmas.

The ELM energy losses in the non-seeded JET-ILW plasmas are smaller than in the JET-C plasmas, both concerning the absolute losses and the relative losses $\left(\Delta \mathrm{W}_{\mathrm{ELM}}^{\mathrm{e}} / \mathrm{W}_{\text {ped }} \approx 4 \%-6 \%\right.$ for the non-seeded JET-ILW and $\approx 6 \%-10 \%$ for the JET-C). So far, JET-ILW plasmas with pedestal heights comparable to the JET-C have been obtained only by seeding nitrogen. In these cases, JET-ILW shots show energy losses similar to the JET-C shots.

The ELM time scale shows a major difference between non-seeded JET-ILW and JET-C as well. First of all, the JET-ILW ELM collapse is often followed by a further slow transport event. The slow transport events are not present in the JET-C and disappear in the $\mathrm{N}_{2}$ seeded JET-ILW shots that have pedestal heights comparable to the JET-C. However, no clear trend between the frequency of these events and the pedestal parameters has been observed. The origin of the slow transport events is still unclear. Second, the ELM time scale is larger in the non-seeded JET-ILW plasmas $\left(\tau_{\mathrm{ELM}}>1.5 \mathrm{~ms}\right)$ than in the JET-C $\left(\tau_{\mathrm{ELM}} \approx 0.6 \mathrm{~ms}\right)$. The seeded JET-ILW plasmas can reach times time scales lower than $1 \mathrm{~ms}$ and comparable to the JET-C ones. 
The difference in ELM energy losses between the non-seeded JET-ILW plasmas and the JET-C plasmas is probably not a direct effect of the difference in the pedestal height. Instead, the low pedestal temperature of the JET-ILW shots is reflected into pedestal collisionality higher than the in JET-C. Due to the high pedestal collisionality, the non-seeded JET-ILW plasmas have energy losses lower than the corresponding JET-C shots. When $\mathrm{N}_{2}$ seeding increases the JET-ILW pedestal temperature to values comparable to the JET-C, a comparable collisionality is achieved. As a consequence, the JET-ILW ELM energy losses are also comparable to those in the JET-C. The pedestal collisionality is well correlated also with the time scale of the ELM collapse. However, the physics reason behind the lower pedestal temperature in the non-seeded JET-ILW and the role of nitrogen in the confinement improvement is still under investigation and it has not been identified yet.

Finally, the presence of the slow transport events could have in principle modified the predictions of the ELM energy losses in ITER. The slow transport events have in fact energy losses twice as large as those obtained in the earlier ELM studies (figure 12). However, the slow transport events disappear in the $\mathrm{N}_{2}$ seeded shots once the JET-ILW recovers a pedestal comparable to the JET-C. This suggests that the slow transport events might be not a major problem for ITER. Excluding the slow transport events, the JET-ILW data fits well within the earlier scaling of the ELM energy losses with the pedestal collisionality.

\section{ACKNOWLEDGEMENT}

This work was supported by EURATOM and carried out within the framework of the European Fusion Development Agreement. The views and opinions expressed herein do not necessarily reflect those of the European Commission.

\section{REFERENCES}

[1] Zhitlukin et al., J. Nucl. Matter 363, 301 (2007)

[2] R.A. Pitts et al., J. Nucl. Mater. 415, S957 (2011).

[3] R.A. Pitts et al., J. Nucl. Mater. 438, S48 (2013).

[4] A. Loarte, G. Saibene, R. Sartori et al., Plasma Phys. Control. Fusion 451549 (2003)

[5] S. Brezinszek et al. 2012 Proc. 24th Int. Conf. on Fusion Energy (San Diego, 2012)

[EX/4-1] http://www-naweb.iaea.org/napc/physics/FEC/FEC2012/index.htm

[6] G. Matthews et al., J. Nucl. Mat. 438, S2 (2013)

[7] R. Neu et al., Phys. Scr. 014038 (2009)

[8] M.N.A. Beurskens, L. Frassinetti, C. Challis et al., Nucl. Fusion Nucl. Fusion 54043001 (2014).

[9] C. Giroud, G.P. Maddison, S. Jachmich et al., Nucl. Fusion 53113025 (2013)

[10] ITER Physics Basis Expert Groups on Confinement and Transport and Confinement Modelling and Database, ITER Physics Basis Editors, Nucl. Fusion 39, 2175 (1999)

[11] I. Nunes et al in Fusion Energy 2010 (Proc. 23rd Int. Conf. Daejeon, 2010) (Vienna: IAEA) CD-ROM file [EXC/p8-03] and http://www-naweb.iaea.org/napc/physics/FEC/FEC2010/index.htm

[12] G. Saibene, R. Sartori, A. Loarte, et al., Plasma Phys. Control. Fusion 441769 (2002)

[13] M.N.A. Beurskens, L. Frassinetti, C. Challis et al., Nucl. Fusion 53013001 (2013)

[14] C. Giroud 2012 Proc. 24th Int. Conf. on Fusion Energy (San Diego, 2012) [EX/P5-30] http://www-naweb.iaea.org/napc/physics/FEC/FEC2012/index.htm

[15] C. Giroud, G. Maddison, K. McCormick et al., Nucl. Fusion 52063022 (2012)

[16] J. Schweinzer, A.C.C. Sips, G. Tardini et al., Nucl. Fusion 51113003 (2011)

[17] M.N.A. Beurskens, J. Schweinzer, C. Angioni et al., Plasma Phys. Controll. Fusion 55, 124043 (2013)

[18] P.A. Schneider, E. Wolfrum, M.G. Dunne et al., Plasma Phys. Controll. Fusion 56, 025011 (2014) 
[19] R. Sartori, G. Saibene, L. D. Horton et al., Plasma Phys. Controll. Fusion 46, 723 (2004)

[20] M.J. Leyland, M.N.A. Beurskens, L. Frassinetti et al., Nucl. Fusion 53083028 (2013).

[21] E. Wolfrum et al., Plasma Phys. Control. Fusion 53085026 (2011)

[22] M.N.A. Beurskens et al. 2012 Proc. 24th Int. Conf. on Fusion Energy (San Diego, 2012) [EX/P7-20] http://www-naweb.iaea.org/napc/physics/FEC/FEC2012/index.htm

[23] E. de la Luna et al., Rev. Sci. Instr. 74, 1414 (2003)

[24] L. Barrera, E. de la Luna, L. Figini et al., Plasma Phys. Controll. Fusion 52, 085010 (2010)

[25] A. Sirinelli, B. Alper, C. Bottereau, et al., Rev. Sci. Instrum. 81, 10D939 (2010)

[26] R. Pasqualotto, P. Nielsen, C. Gowers et al., Rev. Sci. Instrum. 75, 3891 (2004).

[27] L. Frassinetti, M.N.A. Beurskens, R. Scannell et al., Rev. Sci. Instrum. 83, 013506 (2012)

[28] Y. Martin et al., JPCS 123012033 (2008)

[29] A Loarte, M Becoulet, G Saibene, et al., Plasma Phys. Control. Fusion 441815 (2002)

[30] M. N. A. Beurskens, T. H. Osborne, L. D. Horton et al., Plasma Phys. Control. Fusion 51124051 (2009)

[31] M.N.A. Beurskens, A. Alfier, B. Alper et al., Nucl. Fusion 49125006 (2009) 